TRANSACTIONS OF THE

AMERICAN MATHEMATICAL SOCIETY

Volume 357, Number 5, Pages 1693-1715

S 0002-9947(04)03445-2

Article electronically published on December 16, 2004

\title{
A NEW LÖWENHEIM-SKOLEM THEOREM
}

\author{
MATTHEW FOREMAN AND STEVO TODORCEVIC
}

\begin{abstract}
This paper establishes a refinement of the classical LöwenheimSkolem theorem. The main result shows that any first order structure has a countable elementary substructure with strong second order properties. Several consequences for Singular Cardinals Combinatorics are deduced from this.
\end{abstract}

\section{INTRODUCTION}

The Löwenheim-Skolem Theorem [21] is one of the classical and formative results of first order logic. Its consequences have been important technically in the development of Model Theory, Set Theory and interesting from the point of view of the Philosophy of Mathematics [16]. In this paper we improve the Löwenheim-Skolem Theorem and deduce important combinatorial consequences from the stronger version.

There have been many attempts to improve and generalize properties of first order logic to stronger logics. The literature is much too large to survey here. One property that defines first order logic is the fact that every infinite structure in a countable language has a countable elementary substructure. This is not true of many stronger logics such as second order logic. In particular, in the classical Löwenheim-Skolem theorem, one has no control over the second order properties of the elementary substructure.

In this paper we prove that if one fixes in advance a collection of "intervals" around each point in a particular domain $\kappa$ (e.g. a club guessing ladder system), then for all real numbers $r$ and all structures $\mathfrak{A}$ with domain $\kappa$, there is a countable elementary substructure of $\mathfrak{A}$ that has non-empty intersection with exactly those intervals specified by $r$. This is Theorem 3 .

Many of our results will be stated in the language of Stationary Sets. We will use the following definition of stationary: A set $S \subset P(A)$ is stationary iff for all structures $\mathfrak{A}=\left\langle A ; f_{i}, R_{j}, c_{k}\right\rangle_{i, j, k \in \omega}$ in a countable language, there is an elementary substructure $N \prec \mathfrak{A}$ such that $N \in S$. Thus to say that a certain collection of structures is stationary is to assert a type of Löwenheim-Skolem theorem.

The study of stationary sets and their reflection properties has become a central concern of set theory. Stationary set reflection properties often provide combinatorial substitutes for large cardinal reflection properties, while being consistent at small cardinals.

Received by the editors June 26, 2002.

2000 Mathematics Subject Classification. Primary 03C55.

The first author was partially supported by NSF grant DMS-9803126 and the Equipe d'Analyse, Université Paris VI. 
Of particular interest is reflection of stationary families of countable sets. Reflection for these families has many consequences for both combinatorial and descriptive set theory. (See e.g. [6], [24] or [23].)

The structure to which a stationary set reflects is very important in the type of consequence one can deduce. For example, in 23 it is shown that if every stationary set of countable subsets of some $H(\lambda)$ reflects to an internally closed unbounded set, then the singular cardinals hypothesis holds. In 4 it is shown that if every stationary set of countable subsets of some $H(\lambda)$ reflects to a set of uniform cofinality $\omega_{1}$, then there are no very good scales.

This paper gives partial progress towards deciding if ordinary stationary set reflection implies the stronger reflection properties appearing in the theorems mentioned above. Our approach is to postulate ordinary reflection for stationary sets, but to demand reflection for more than one stationary set at a time. From this type of hypothesis we are able to deduce the consequences of stronger reflection mentioned above (and more) and in certain circumstances prove reflection to internally approachable sets.

We now make the definitions necessary to formulate the new results precisely.

Definition 1. Let $\kappa$ be a regular cardinal. A ladder system on a stationary set $S \subset \kappa$ (consisting of ordinals of countable cofinality) is a collection $\left\langle\left\{\delta_{n}: n \in \omega\right\}\right.$ : $\delta \in S\rangle$ with the property that each sequence $\left\{\delta_{n}: n \in \omega\right\}$ is increasing and cofinal in $\delta$. Such a ladder system is called club guessing if for all closed unbounded sets $D \subset \kappa$ there is a $\delta \in D$ with $\left\{\delta_{n}: n \in \omega\right\} \subset D$.

Given a ladder system $\vec{l}(\kappa)$ (which we assume to be club guessing) and a countable subset $N$ of $\kappa$ we can associate an infinite subset of $\omega$, by considering the supremum $\delta$ of $N$ and asking which intervals $\left[\delta_{n}, \delta_{n+1}\right.$ ) have non-empty intersection with $N$.

The classical Löwenheim-Skolem Theorem asserts that for any structure on a cardinal $\kappa$, there is a countable elementary substructure but gives no information (other than that definable in the structure) about where that elementary substructure lives. Our version gives control over which intervals the elementary substructure hits. Precisely:

Definition 2. For $N \subset H(\theta)$ with $N \cap \kappa$ having countable cofinality, the pattern of $N$ at $\kappa$ is defined to be:

$$
\operatorname{pat}(N, \kappa)=\left\{n: N \cap\left[\delta_{n}, \delta_{n+1}\right) \neq \emptyset\right\}
$$

where $\left\langle\delta_{n}: n \in \omega\right\rangle$ is the element of the ladder system for $\kappa$ at $\delta=\sup (N \cap \kappa)$.

Let $\theta \geq \omega_{2}$ be a regular cardinal. Let

$$
P:\left\{\kappa: \omega_{2} \leq \kappa \leq \theta \text { and } \kappa \text { is regular }\right\} \rightarrow[\omega]^{\aleph_{0}} .
$$

The strengthened Löwenheim-Skolem theorem says:

Theorem 3. Let $\mathfrak{A}$ be a structure whose domain is some uncountable regular cardinal. Then there is a countable $N \prec \mathfrak{A}$ such that pat $(N, \kappa)=P(\kappa)$, for all regular $\kappa \in N \cup\{\theta\}$ that are at least $\omega_{2}$.

(In the reformulation (Theorem 15) proved in section 3 we define

$$
S_{P}=\left\{N \in[\theta]^{\aleph_{0}}: \operatorname{pat}(N, \kappa)=P(\kappa) \text {, for all regular } \kappa \in N \cup\{\theta\}\right\}
$$

and restate Theorem 3 as stating that $S_{P}$ is stationary.) 
One may question what this has to do with reflection. The next observation is one of the main tools of this paper:

Lemma 4. Suppose that $X$ is an uncountable set and $X \cap \kappa$ has cofinality $\omega$, and pattern $r \subset \omega$. Then there is a closed unbounded subset $C$ of $[X]^{\aleph_{0}}$ of $N$ that have pattern $r$ at $\kappa$.

Restating this lemma in a negative form, it is impossible for the collection of $N$ that have pattern different from $r$ to reflect to $X$. Thus if $X$ reflects two different patterns at $\kappa$ it must have cofinality $\omega_{1}$.

In particular Lemma 4 together with Theorem 15 implies that there are two stationary sets such that any $X \prec H(\lambda)$ with $|X|=\omega_{1} \subset X$ that reflects both sets must have the property that the cofinality of $X \cap \kappa$ is uncountable for all regular cardinals $\kappa \leq \theta$. This gives as a consequence that simultaneous reflection of any 3 stationary sets gives reflection of stationary sets to sets of size and uniform cofinality $\omega_{1}$. (See Corollary 20) In particular, we can apply the theorems of 4 to see that there are no very good scales.

We explore a concept introduced in [8], that of tightness of a structure. Tightness, per se, is a condition on an uncountable set $X$ relative to a scale. Namely it says that the elements of the scale that are indexed by ordinals in $X$ are cofinal below the characteristic function of $X$. For uncountable structures the stationary set of tight structures can be defined independently of the choice of scale. This condition follows if $X$ is internally approachable, but is not equivalent. (Precise definitions are given later in this section. See the forthcoming [5] for a study of the relationship betwen tightness and approachability.)

For countable structures, it is not completely clear how to define an analogous notion of tight. For the purposes of studying the singular cardinals hypothesis we introduce the notion of weak tightness and show that if the SCH fails the collection of weakly tight structures is stationary. Any uncountable $X$ that reflects the collection of weakly tight structures and is of uniform cofinality $\omega_{1}$ must be tight. As a result of this analysis we are able to deduce the following result:

Theorem 5. Suppose that for all uncountable $\lambda$, every pair of stationary subsets of $[\lambda]^{\aleph_{0}}$ simultaneously reflects to a set of size $\omega_{1}$. Then the singular cardinals hypothesis holds.

In the last section of the paper we assume the existence of partial square sequences on successors of singular cardinals. The existence of such sequences is provable in ZFC for successors of regular cardinals and conjectured to be a theorem for successors of singular cardinals (19]). With this assumption and the assumption that every collection of 4 stationary sets simultaneously reflects we are able to prove that every stationary subset of a $[\kappa]^{\aleph_{0}}$ for $\kappa$ below the first fixed point of the $\aleph$ sequence reflects to a set $X$ of cardinality $\omega_{1}$ with $\omega_{1} \subset X$ such that $X \cap[X]^{<\aleph_{0}}$ is stationary.

Notation and background information. We will attempt to keep our notation as standard as possible. We will follow the custom established by Shelah, of writing $H(\lambda)$ for the sets of hereditary cardinality less than $\lambda$. We will systematically confuse $H(\lambda)$ with a structure of the form $\langle H(\lambda), \in, \Delta\rangle$ where $\Delta$ is a well ordering of $H(\lambda)$. The cardinal $\lambda$ will always be taken to be a "large enough regular cardinal" to reflect any statement we are interested in. We will often also assume that we 
have predicates in the structure for all of the mathematical objects relevant to the context.

We will use standard terminology with respect to filters and their duals, ideals. A set $X$ will have "measure zero" with respect to an ideal $I$ iff $X \in I$; it will have "measure one" iff $X \in F$, where $F$ is the dual ideal. If $X$ does not have measure zero, then $X$ is $I$-positive $\left(X \in I^{+}\right)$.

We will frequently be using the following theorem of Shelah:

Theorem ([18]). Suppose that $\kappa \geq \omega_{2}$ is a regular cardinal. Then for all stationary $T \subset \kappa$ consisting of singular ordinals, there is a stationary set $S \subset T$ and a club guessing sequence defined on $S$.

(In particular there are stationary $S \subset \kappa \cap \operatorname{cof}(\omega)$ on which there are club guessing ladder systems.)

Definition 6. Suppose that $\vec{l}(\kappa)$ is a club guessing ladder system on $S \subset \kappa$, a regular cardinal. We define two filters. The Club Guessing Filter is the filter generated by sets of the form $X_{D}=\left\{\delta \in S:\left\{\delta_{n}: n \in \omega\right\} \subset D\right\}$, for closed unbounded sets $D \subset \kappa$. The Weak Club Guessing Filter is the filter generated by the sets $Y_{D}=\left\{\delta \in S\right.$ : for all large enough $\left.n, \delta_{n} \in D\right\}$ for closed unbounded sets $D$.

Both filters are $\kappa$-complete proper filters, but (as remarked by Ishiu) the club guessing filter is never normal (its normal closure is not a proper filter). The weak club guessing filter is always normal.

Definition 7. Suppose that $B \subset A$. Then a set $S \subset P(A)$ is said to reflect to $B$ iff $S \cap P(B)$ is stationary. We say that stationary sets $\left\{S_{i}: i \in I\right\}$ simultaneously reflect to $B$ iff for all $i \in B \cap I, S_{i} \cap P(B)$ is stationary.

We will be interested in variations of the following weak reflection property:

Suppose that $S \subset[H(\lambda)]^{\aleph_{0}}$ is stationary. Then there is a set $X$ having cardinality $\aleph_{1}$ with $\omega_{1} \subset X$ such that $S$ reflects to $X$.

We will say (loosely) that $S$ reflects to a set of size $\omega_{1}$. The requirement that $\omega_{1} \subset X$ is to separate reflection from issues involving Chang's Conjecture (see [7]) but its role is not entirely understood. (See Problem 9 at the end of the paper.)

One thrust of this paper is to study what structure we can assume $X$ has. Some properties of $X$ we can consider:

- $X$ is internally unbounded iff $X \cap[X]^{\aleph_{0}}$ is unbounded.

- $X$ is internally stationary iff $X \cap[X]^{\aleph_{0}}$ is stationary.

- $X$ is internally club iff $X \cap[X]^{\aleph_{0}}$ is closed and unbounded.

- $X$ is internally approachable. (Here we will take this to mean that there is a sequence $\left\langle X_{\alpha}: \alpha<|X|\right\rangle$ that is continuous, increasing with $\alpha$, has union $X$ and is such that $\left|X_{\alpha}\right|<|X|$ and every initial segment of the sequence belongs to $X$. See [9] for more information about internally approachable structures.)

These properties are clearly ascending in strength and equivalent (for typical uncountable $X$ ) under $\mathrm{CH}$. However their relationship without $\mathrm{CH}$ is unknown. (See Problem (7) A related property of an $X \prec H(\lambda)$ is that it has uniform cofinality $\rho$ on some collection $K$ of cardinals. This means that for all $\kappa \in K, \operatorname{cof}(X \cap \kappa)=\rho$. 
Clearly if $X$ has cardinality $\omega_{1}$, then any of the properties above implies that $X$ has uniform cofinality $\omega_{1}$ in all regular cardinals $\kappa$.

We now see many variants on the weak reflection property of the form:

Every stationary set (collection of stationary sets) with property

$\mathrm{P}$ reflects (simultaneously reflects) to a set with property $\mathrm{Q}$.

In particular we will use the phrase "any collection of $\kappa$ stationary sets reflects to a set of size $\omega_{1}$ " to mean that there is an $X$ with $|X|=\omega_{1}, \omega_{1} \subset X$ such that $X$ simultaneously reflects the collection of stationary sets.

The main theme of this paper is that one can replace hypotheses of the form "any stationary set can be reflected to a set with property Q", by statements of the form "any $\kappa$ stationary sets can be simultaneously reflected to a set of size $\omega_{1}$ " (where $\kappa$ is a small cardinal such as 2, 3 or 4 ) and get similar consequences.

We now make some basic observations about reflection.

Lemma 8. Suppose that every stationary set $S \subset[H(\lambda)]^{\aleph_{0}}$ reflects to sets of size $\omega_{1}$. Then for any stationary set $S \subset[H(\lambda)]^{\aleph_{0}}$ the collection of $X \in[H(\lambda)]^{\aleph_{1}}$ to which $S$ reflects is stationary.

Proof. Let $\mathfrak{A}$ be a structure with domain $H(\lambda)$. Let $X$ reflect $\{N \in S: N \prec \mathfrak{A}\}$. Then $X \prec \mathfrak{A}$.

The converse of this is also true:

Lemma 9. Suppose that $S \subset[H(\lambda)]^{\aleph_{0}}$ and $\left\{X \in[H(\lambda)]^{\aleph_{1}}: S \cap[X]^{\aleph_{0}}\right.$ is stationary $\}$ is stationary. Then $S$ is stationary.

Proof. Let $\mathfrak{A}$ be a structure with domain $H(\lambda)$. Let $X \prec \mathfrak{A}$ reflect $\{N \in S: N \prec$ $\mathfrak{A}\}$. Then there is an $N \in S \cap[X]^{\aleph_{0}}, N \prec X$, hence $S$ is stationary.

We now remark on a standard trick:

Lemma 10. Let $\mathfrak{A}$ be an expansion of $\langle H(\lambda), \in, \Delta\rangle$ for a large regular $\lambda$. Suppose that $N \prec \mathfrak{A}$ and $x, \kappa \in N$ and $|x|<\kappa$. Let $M=s k^{\mathfrak{A}}(N \cup x)$. Then:

$$
\sup (N \cap \kappa)=\sup (M \cap \kappa) .
$$

Proof. Every element of $M$ is of the form $\tau(a, y)$ where $a \in N$ and $y \in x$ for $\tau$ a definable Skolem function in $\mathfrak{A}$. Since $|x|<\kappa$ and $x, \kappa \in N$, we have $N \models$ $\sup \{\tau(a, y): y \in x\}<\kappa$ and for some $\gamma \in N \cap \kappa, \gamma=\sup \{\tau(a, y): y \in x\}$.

Definitions of PCF objects. We refer the daring reader to [18] for information on PCF theory. Those more easily satisfied might find [3] or [12] easier reading.

Reduced products have been used to study singular cardinals by many authors (see [20], [11]). Shelah developed an extensive theory of these, notably at cardinals of countable cofinality.

Typically the PCF theory studies a singular cardinal $\kappa$ by considering an increasing cofinal sequence of regular cardinals $\left\langle\kappa_{i}: i \in c f(\kappa)\right\rangle$, and an ideal $I$ on $c f(\kappa)$. Then properties of the reduced product $\prod_{i \in c f(\kappa)} \kappa_{i} / I$ are used to bootstrap properties of the $\kappa_{i}$ to $\kappa^{+}$. In this paper we will always make the harmless assumption that all of the $\kappa_{i}$ are at least $\omega_{2}$.

We recall that the partial ordering on the reduced product is defined as follows:

Let $f, g \in \prod_{i \in c f(\kappa)}$

- We put $f=_{I} g$ iff $\{i: f(i) \neq g(i)\} \in I$. 
- We put $f<_{I} g$ (or $f \leq_{I} g$ ) iff $\{i: f(i) \geq g(i)\} \in I$ (resp: $\{i: f(i)>g(i)\} \in$ $I)$.

(If $I$ is the ideal of bounded sets, then we will write $f={ }^{*} g$ for $f={ }_{I} g$ and $f<{ }^{*} g$ for $f<_{I} g$ etc. In this case if for all $i>k, f\left(\kappa_{i}\right)<g\left(\kappa_{i}\right)$, we will write $f<_{k} g$.)

A scale in $\prod_{i \in c f(\kappa)} \kappa_{i} / I$ is a sequence $\left\{f_{\alpha}: \alpha<\lambda\right\}$ such that:

(1) $\alpha<\beta<\lambda$ implies $f_{\alpha}<_{I} f_{\beta}$.

(2) For all $f \in \prod_{i \in c f(\kappa)} \kappa_{i} / I$ there is an $\alpha<\lambda, f<_{I} f_{\alpha}$.

If $\left\{f_{\alpha}: \alpha<\gamma\right\}$ is a scale, then any cofinal subsequence is a scale and hence we can assume that $\lambda$ is always a regular cardinal. In this case we will say that the true cofinality of $\prod_{i \in c f(\kappa)} \kappa_{i} / I$ is $\lambda$. (We note that this is well defined.)

Shelah has shown that there is always a sequence of cardinals $\kappa_{i}$ such that $\prod_{i \in c f(\kappa)} \kappa_{i} / I$ has a scale of length $\kappa^{+}$, where $I$ is the ideal of bounded subsets of $c f(\kappa)$.

An exact upper bound for a sequence $\left\langle f_{\alpha}: \alpha<\gamma\right\rangle \subset \prod \kappa_{i}$ is a function $g$ such that if $h<_{I} g$, then there is an $\alpha<\gamma$ such that $f<_{I} f_{\alpha}$. If an exact upper bound exists it is clearly unique $(\bmod I)$. We will assume that our scales are continuous in the sense that if $\left\langle f_{\alpha}: \alpha<\gamma\right\rangle$ has an exact upper bound, then $f_{\gamma}$ is the exact upper bound.

If $\left\langle f_{\alpha}: \alpha<\lambda\right\rangle$ is a continuous $<_{I}$ increasing sequence of functions, then $\gamma$ is a good point for the sequence iff there is a sequence of functions $\left\langle g_{\delta}: \delta \in c f(\gamma)\right\rangle$ of length $c f(\gamma)$ which is pointwise increasing on a fixed $I$-large subset of $c f(\kappa)$ and is cofinally interleaved with $\left\langle f_{\alpha}: \alpha<\gamma\right\rangle$ modulo $I$; i.e. that for all $\alpha<\gamma$ there are $\delta<c f(\gamma), \alpha^{\prime}<\gamma$ such that $f_{\alpha}<_{I} g_{\delta}<_{I} f_{\alpha^{\prime}}$. If $I$ is the ideal of bounded subsets of $c f(\kappa)$, then this is equivalence to the existence of an unbounded set $A \subset \gamma$ and a set $B \in I$ such that for all $\kappa_{i} \notin B,\left\{f_{\alpha}\left(\kappa_{i}\right): \alpha \in A\right\}$ is strictly increasing. It is a theorem of Shelah that for ordinals $\gamma$ of cofinality larger than $c f(\kappa), \gamma$ is a good point iff there is an exact upper bound $g$ for $\left\langle f_{\alpha}: \alpha<\gamma\right\rangle$ with $\operatorname{cf}\left(g\left(\kappa_{i}\right)\right)=c f(\gamma)$ for almost all $\kappa_{i}$. In particular the collection of good points is stationary. A point $\gamma$ is promising iff $A$ can be taken to be stationary in $\gamma$, and very good iff $A$ can be taken to be closed and unbounded. A point $\gamma$ is better iff there is a closed unbounded set $A \subset \gamma$ such that for all $\beta \in A$, there is a $B \in I$ such that for all $\kappa_{i} \notin B,\left\{f_{\alpha}\left(\kappa_{i}\right): \alpha \in A \cap \beta\right\}$ is strictly increasing. Clearly, if $\gamma$ is very good, then it is better, and better implies promising.

Easy arguments show that if $\gamma$ is a good point and $E \subset \gamma$ is unbounded, there is an unbounded subset $E^{\prime} \subset E$ such that $E^{\prime}$ satisfies the definition of good. It is also easy to show that if $\gamma$ is a good point of cofinality at least $c f(\kappa)^{+2}$, then $\gamma$ is promising.

A scale will be called good (resp. very good, better) iff there is a closed unbounded $D \subset \lambda$ such that every $\gamma \in D$ with cofinality greater than $c f(\kappa)$ is good (resp. very good, better).

Shelah has shown that if the SCH fails first at a cardinal $\kappa$, then there is a cofinal sequence $\left\langle\kappa_{n}: n \in \omega\right\rangle$ in and a better scale on $\prod \kappa_{n} / I$, where $I$ is the ideal of bounded sets.

Good, better and very good scales and their relations with reflection properties were studied systematically in [4], to which we refer the reader for information. 
We finish with two important definitions.

Definition 11. Let $K$ be a set of cardinals and $N$ a set. The characteristic function of $N$ (denoted $\chi_{N}$ ) is defined to be $\chi_{N}(\kappa)=\sup (N \cap \kappa)$ for $\kappa \in K$. In contexts where we are working with a scale on $\prod \kappa_{i}$ for some sequence of cardinals $\left\langle\kappa_{i}\right\rangle$, we will take $K$ to be the collection of $\kappa_{i}$ 's.

If we fix a sequence $\vec{\kappa}=\left\langle\kappa_{i}\right\rangle$ and an ideal $I$ on $\left\{\kappa_{i}\right\}$ we will say that $N$ is tight for $\vec{\kappa}$ and $I$ iff $N \cap \prod \kappa_{i}$ is cofinal in $\prod\left(N \cap \kappa_{i}\right)$ modulo $<_{I}$.

If we have a scale $\left\langle f_{\alpha}: \alpha<\lambda\right\rangle \subset \prod \kappa_{i} / I$ and $N \prec\left\langle H(\lambda), \in, \Delta,\left\langle f_{\alpha}: \alpha<\lambda\right\rangle\right\rangle$, then tightness is equivalent to the statement that $\chi_{N}$ is an exact upper bound for $\left\{f_{\alpha}: \alpha \in N \cap \lambda\right\} \bmod I$.

In our context we will always be working with tight structures of a uniform cofinality; however tight does not imply uniform cofinality (see [5]).

\section{Standard NAmba LEMmas}

In this section we quote some useful results about "Namba Combinatorics" that are well known. (The reader is referred to [14, 13, 11, [17, [2, [15]) for the mathematical and historical background of this area.)

The basic objects for this theory are trees that split into positive sets relative to certain (proper) filters. The relevant property of these filters is their completeness.

Let $\mathcal{T} \subset[O R]^{<\omega}$ be a tree and suppose that for each $\sigma \in \mathcal{T}$ we have a filter $F_{\sigma}$ on $\{\alpha: \sigma \frown \alpha \in \mathcal{T}\}$. Let $c(\sigma)$ be the completeness of this filter.

Definition 12. Given a tree $\mathcal{T} \subset[O R]^{<\omega}$, the stem of $\mathcal{T}$ is the collection of $\sigma \in \mathcal{T}$ such that for all $\tau \in \mathcal{T}$ either $\sigma \subset \tau$ or $\tau \subset \sigma$. All of our trees will have a finite stem. A Namba subtree of $\mathcal{T}$ is a subtree $\mathcal{T}^{\prime} \subset \mathcal{T}$ such that for all $\sigma \in \mathcal{T}^{\prime}$ not lying in a proper initial segment of the stem of $\mathcal{T}^{\prime},\left\{\alpha: \sigma^{\frown} \alpha \in \mathcal{T}^{\prime}\right\}$ is positive for the filter $F_{\sigma}$.

Example 13 (Namba). Let $\mathcal{T}$ be a subtree of $\omega_{2}^{<\omega}$ such that every node $\sigma$ not in a proper initial segment of the stem of $\mathcal{T}$ is $\omega_{2}$ splitting (i.e. $|\{\alpha: \sigma \frown \alpha \in \mathcal{T}\}|=\omega_{2}$ ). For each $\sigma$ not in a proper initial segment of the stem of $\mathcal{T}$ let $F_{\sigma}$ be the filter of cobounded subsets of $\omega_{2}$.

This example is fairly typical in that the filters for a Namba tree are usually a variation on either the filter of cobounded sets or the filter of closed unbounded sets. (Namba considered similar forcings on different cardinals in his papers.)

Recall that if $\mathcal{T}$ is a tree there is a topology on the collection of all branches through $\mathcal{T}$ (which we will denote by $[\mathcal{T}]$ ) obtained by taking the topology induced by the product topology on $\kappa^{\omega}$ for some $\kappa$ larger than the supremum of all ordinals appearing in a sequence in $\mathcal{T}$.

The basic Namba lemma appears in [14] among other places:

Theorem 14. Suppose that $\mathcal{T}$ is a Namba tree such that for all $\sigma \in \mathcal{T}, c(\sigma)>\kappa$. Suppose that

$$
[\mathcal{T}]=\bigcup_{i<\kappa} X_{i}
$$

where each $X_{i}$ is a Borel subset of $[\mathcal{T}]$. Then there is a Namba subtree $\mathcal{T}^{\prime} \subset \mathcal{T}$ such that for some $i<\kappa,\left[\mathcal{T}^{\prime}\right] \subset X_{i}$.

The fact that each $X_{i}$ is Borel is used to see that a particular game is Borel and hence determined. In most applications, the sets $X_{i}$ will be closed sets. 


\section{A strong LöWenheim-Skolem Theorem}

In this section we give the proof of a strong Löwenheim-Skolem Theorem.

We begin by restating the data for the theorem. Let $\theta$ be a regular cardinal, and $P:\left\{\kappa: \omega_{2} \leq \kappa \leq \theta\right.$ and $\kappa$ is regular $\} \rightarrow[\omega]^{\aleph_{0}}$ a function. Fix for each regular cardinal $\omega_{2} \leq \kappa \leq \theta$ a club guessing ladder system $\vec{l}(\kappa)$.

Recall that for $N \subset H(\theta)$ with $N \cap \kappa$ having countable cofinality, the pattern of $N$ at $\kappa$ is defined to be:

$$
\operatorname{pat}(N, \kappa)=\left\{n: N \cap\left[\delta_{n}, \delta_{n+1}\right) \neq \emptyset\right\}
$$

where $\left\langle\delta_{n}: n \in \omega\right\rangle$ is the element of the ladder system for $\kappa$ at $\delta=\sup (N \cap \kappa)$. Using this data we can define the following set:

$$
S_{P}=\left\{N \in[H(\theta)]^{\aleph_{0}}: \operatorname{pat}(N, \kappa)=P(\kappa) \text {, for all } \kappa \in N \cap \operatorname{dom}(P)\right\} .
$$

The main theorem of this section is:

Theorem 15. $S_{P}$ is stationary.

Proof. Fix an arbitrary structure $\mathfrak{A}=\langle H(\xi), \Delta, \theta, \vec{l}, P, \ldots\rangle$ for a regular $\xi>\theta$. We need to find a countable elementary substructure $N \prec \mathfrak{A}$ such that for all $\kappa \in N \cap \operatorname{dom}(P), \operatorname{pat}(N, \kappa)=P(\kappa)$.

To see the result we build a $\subset$-decreasing sequence of Namba-trees (in the appropriate sense) $\left\langle\mathcal{T}_{n}: n \in \omega\right\rangle$ with the property that if $b$ is a branch through all of the $\mathcal{T}_{n}$, then $N=s k^{\mathfrak{A}}(b)$ has the desired property.

Each tree $\mathcal{T}_{n}$ will be a subset of $\theta^{<\omega}$. The nodes $\sigma \in \mathcal{T}_{n}$ will have a label $s(\sigma)$ among the regular cardinals less than or equal to $\theta$. The trees $\mathcal{T}_{n}$ will be Namba in the sense that there will be finitely many regular cardinals $\left\{\kappa_{0}, \ldots, \kappa_{n-1}\right\}$ less than or equal to $\theta$ such that:

- If $s(\sigma)=\kappa_{i}$ for some $i$, then there is a unique $\alpha<\kappa_{i}$ such that $s(\sigma)^{\frown} \alpha \in \mathcal{T}_{n}$.

- If $s(\sigma)=\kappa \notin\left\{\kappa_{0}, \ldots, \kappa_{n-1}\right\}$, then $\left\{\alpha: \sigma \frown \alpha \in \mathcal{T}_{n}\right\} \subset \kappa$ is unbounded in $\kappa$.

We require that the labelling $s$ will have the following two properties:

(1) If $b$ is a branch through $\mathcal{T}_{0}$ and $\sigma=b\left\lceil k\right.$ for some $k$ and $\kappa \in s k^{\mathfrak{A}}(\sigma)$ is regular, then there is an infinite set of $m \geq k$ such that every $\tau \in \mathcal{T}_{n}$ with $\sigma \subset \tau$ and $|\tau|=m$ has $s(\tau)=\kappa$.

(2) If $s(\tau)=\kappa$, then $\kappa \in \operatorname{sk}(\tau)$.

The $\mathcal{T}_{n}$ 's will also have the property that there are $\delta_{0}, \ldots, \delta_{n-1} \in O R$ such that for all branches $b$ through $\mathcal{T}_{n}$,

- $\sup \left(s k^{\mathfrak{A}}(b) \cap \kappa_{i}\right)=\delta_{i}$.

- $\kappa_{i} \in s k^{\mathfrak{A}}(b)$.

- $s k^{\mathfrak{A}}(b)$ has the pattern $P\left(\kappa_{i}\right)$ at $\kappa_{i}$.

We will say that a $\mathcal{T}_{n}$ with these properties is acceptable for this sequence $\left\{\kappa_{0}, \ldots, \kappa_{n-1}\right\}$, and dually that each $\kappa_{i}$ is fixed for $\mathcal{T}_{n}$.

By carefully enumerating the $\kappa_{i}$ 's in the Skolem hulls of the stems of the various $\mathcal{T}_{n}$, it suffices to show the following lemma:

Lemma 16. Suppose that $\mathcal{T}$ is a Namba tree acceptable for $\left\{\kappa_{0}, \ldots, \kappa_{n-1}\right\}$ and $\kappa \leq \theta$ is in $s k^{\mathfrak{A}}(\sigma)$, where $\sigma$ is the stem of $\mathcal{T}$. Then there is a $\mathcal{T}^{\prime} \subset \mathcal{T}$ that is acceptable for $\left\{\kappa_{0}, \ldots, \kappa_{n-1}, \kappa\right\}$. 
To prove this lemma, we build intermediate subtrees of $\mathcal{T}$ and utilize these to get $\mathcal{T}^{\prime}$. All of the trees will be the result of judicious pruning by strategies (in two different games).

3.1. The first game. In the first game $\mathcal{G}^{1}$ players $I$ and $I I$ cooperate to build a branch through $\mathcal{T}$ according to the following schedule. At a stage where the sequence $\sigma \in \mathcal{T}$ has been constructed:

(1) If $s(\sigma)<\kappa$ (or $s(\sigma) \in\left\{\kappa_{0}, \ldots, \kappa_{n-1}\right\}$ ), player II plays an ordinal $\alpha<s(\sigma)$ such that $\sigma^{\frown} \alpha \in \mathcal{T}$.

(2) If $s(\sigma)>\kappa$ and $s(\sigma) \notin\left\{\kappa_{0}, \ldots, \kappa_{n-1}\right\}, I I$ chooses an ordinal $\gamma<s(\sigma)$ and $I$ chooses an $\alpha$ with $\gamma<\alpha<s(\sigma)$ such that $\sigma^{\frown} \alpha \in \mathcal{T}$.

(3) At stages $\sigma$ where $s(\sigma)=\kappa$ player $I$ plays an ordinal $\beta, I I$ plays an ordinal $\gamma>\beta$ and $I$ plays an $\alpha>\gamma$ with $\sigma^{\frown} \alpha \in \mathcal{T}$.

A play of the game results in:

- a branch $b$ through the tree $\mathcal{T}$;

- a sequence $\left\langle\beta_{n}: n \in S \subset \omega\right\rangle$ of ordinals played by player $I$ at stages $\sigma$ with $s(\sigma)=\kappa$

- a sequence of ordinals $\left\langle\gamma_{n}: n \in S\right\rangle$ played by $I I$ at the same stages. Note that for all $n, \beta_{n}<\gamma_{n}$.

\section{The winning condition:}

Player $I$ wins the game $\mathcal{G}^{1}$ iff:

$$
s k^{\mathfrak{A}}(b) \cap \bigcup_{n \in S}\left[\beta_{n}, \gamma_{n}\right)=\emptyset .
$$

Note. While the game $\mathcal{G}^{1}$ is closed (and hence determined), this fact appears not to be relevant to finding a strategy for $I$.

Claim 17. There is a tree $\mathcal{T}^{*} \subset \mathcal{T}$ that is acceptable for $\left\{\kappa_{0}, \ldots, \kappa_{n-1}\right\}$ such that Player I has a winning strategy in $\mathcal{G}^{1}$ played on $\mathcal{T}^{*}$.

Proof. To see this, note that by reducing $\mathcal{T}$ somewhat and (possibly) adding functions to the type of $\mathfrak{A}$ we can assume that for all $\sigma \in \mathcal{T}$ with $s(\sigma)=\kappa$, there is an $f_{\sigma}$ definable in $\mathfrak{A}$ from $\sigma$ such that

$$
f_{\sigma}: \kappa \cap \operatorname{cof}\left(\omega_{1}\right) \rightarrow\left\{\alpha: \sigma^{\frown} \alpha \in \mathcal{T}\right\}
$$

is a bijection with $f_{\sigma}(\delta) \geq \delta$ for all $\delta$. Since expanding a structure increases the Skolem hull, it suffices to show the claim for the expanded structure.

Note that if $b$ is a branch through $\mathcal{T}$ and $c$ is the result of replacing each $\alpha=b(n)$ arising from a stage $\sigma=b\left\lceil n\right.$ with $s(\sigma)=\kappa$ by $f_{\sigma}^{-1}(\alpha)$, then

$$
s k^{\mathfrak{A}}(b)=s k^{\mathfrak{A}}(c) \text {. }
$$

Thus, as far as Skolem hulls go, we can assume that for all stages $\sigma$ with $s(\sigma)=\kappa$ :

$$
\{\alpha: \sigma \frown \alpha \in \mathcal{T}\}=\kappa \cap \operatorname{cof}\left(\omega_{1}\right) .
$$

We now make this assumption replacing $\mathcal{T}$ for the moment by the "isomorphic" $\mathcal{S}$.

For each $\alpha \in \kappa \cap \operatorname{cof}\left(\omega_{1}\right)$, we choose $\left\langle\alpha(i): i \in \omega_{1}\right\rangle$ converging to $\alpha$. For each infinite branch $c=\left\langle\alpha_{n}: n \in \omega\right\rangle$ through $\mathcal{S}$ and each $n$ with $s(c \mid n)=\kappa$, let $i_{n}^{c}$ be the least $i \in \omega_{1}$ such that $\alpha_{n}(i)>s k^{\mathfrak{A}}(c) \cap \alpha_{n}$. Let $i(c)=\sup \left\langle i_{n}^{c}: n \in \omega\right\rangle$. 
By Theorem 14 we see that there is a tree $\mathcal{S}^{*} \subset \mathcal{S}$ and a $\beta^{*}<\omega_{1}$ such that:

- For all $\sigma \in \mathcal{S}^{*}$, if $s(\sigma) \neq \kappa_{l}$ for any $l<n$ then $\sigma$ is an $s(\sigma)$ splitting node of $\mathcal{S}^{\prime}$.

- If $s(\sigma)=\kappa$ then for stationarily many $\alpha<\kappa, \sigma^{\frown} \alpha \in \mathcal{S}^{*}$.

- For all branches $c$ through $\mathcal{S}^{*}, i(c)=\beta^{*}$.

We let $\mathcal{T}^{*}$ be the subtree of $\mathcal{T}$ corresponding to $\mathcal{S}^{*}$.

To see that $\mathcal{T}^{*}$ satisfies the claim, we define a winning strategy for player $I$.

We describe her plays on $\mathcal{S}^{*}$ rather than $\mathcal{T}^{*}$ for convenience. Note that player $I$ has discretion to play only at $\sigma$ with $s(\sigma) \geq \kappa$.

By cases:

- At stages $\sigma$ where $s(\sigma)>\kappa$, player $I$ plays arbitrarily according to the rules of $\mathcal{G}^{1}$.

- At stages $\sigma$ where $s(\sigma)=\kappa$, consider the map from $X=\left\{\alpha: \sigma \frown \alpha \in \mathcal{S}^{*}\right\}$ to $\kappa$ given by $\alpha \mapsto \alpha\left(\beta^{*}\right)$. This function is regressive, and hence constantly $\beta_{\sigma}$ on a stationary subset $X^{\prime} \subset X$ for some $\beta_{\sigma}$. Player $I$ now plays $\beta_{\sigma}$ and when $I I$ responds with some $\gamma$, player $I$ plays an $\alpha \in X^{\prime} \backslash \gamma$.

To see that this is a winning strategy on $\mathcal{T}^{*}$ we note that if $c$ is the result of playing according to this strategy, then for all $n$ with $\sigma=c\lceil n$ and $s(\sigma)=\kappa$ we have that $s k^{\mathfrak{A}}(c) \cap c(n) \subset \beta_{\sigma}=\beta_{n}$. Since $\beta_{n}<\gamma_{n}<c(n)$, we have $s k^{\mathfrak{A}}(c) \cap\left[\beta_{n}, \gamma_{n}\right)=\emptyset$. By the definability and monotonicity of the $f_{\sigma}$ 's we have $c(n) \leq b(n)$ and $s k^{\mathfrak{A}}(c)=$ $s k^{\mathfrak{A}}(b)$ for the branch $b$ through $\mathcal{T}^{*}$ corresponding to $c$. This proves the claim.

Claim 18. There is a tree $\mathcal{T}^{\dagger} \subset \mathcal{T}^{*}$ that is acceptable for $\left\{\kappa_{0}, \ldots, \kappa_{n-1}\right\}$ such that every branch b through $\mathcal{T}^{\dagger}$ is the result of a play of the game $\mathcal{G}^{1}$ according to $\mathcal{W}$, where $\mathcal{W}$ is I's winning strategy in $\mathcal{G}^{1}$.

Proof. This claim is standard so we sketch the proof. Decide which elements $\sigma$ of $\mathcal{T}^{*}$ to put in $\mathcal{T}^{\dagger}$ by induction on $n$. In the process define a partial play of the game $\mathcal{G}^{1}$ according to $\mathcal{W}$ to associate with $\sigma$. At any inning, a play by $I I$ is canonically associated with an ordinal, and hence canonically well ordered.

For $s(\sigma)<\kappa$, put all one point extensions of $\sigma$ into $\mathcal{T}^{\dagger}$, since they all canonically correspond to a legal play by player $I I$.

For $s(\sigma) \geq \kappa$, put every $\tau$ into $\mathcal{T}^{\dagger}$ that is a one point extension of $\sigma$ corresponding to a response by $\mathcal{W}$ to some play by $I I$ in the the partial play of the game associated with $\sigma$. Associate to each $\tau$ the least such play by $I I$ eliciting this response, together with its response by $\mathcal{W}$.

3.2. The second game. Define a game $\mathcal{G}_{\delta}^{2}$ for those $\delta \in \kappa \cap \operatorname{cof}(\omega)$ where the ladder system $\vec{l}(\kappa)$ is defined. For these $\delta$ let $\left\langle\delta_{n}: n \in \omega\right\rangle$ be $\vec{l}(\kappa)$ at $\delta$. In $\mathcal{G}_{\delta}^{2}$ players $I$ and $I I$ cooperate to produce a play of the game $\mathcal{G}^{1}$ by producing a branch through $\mathcal{T}^{\dagger}$.

At a stage $\sigma \in \mathcal{T}^{\dagger}$ :

- If $s(\sigma)<\kappa$, player $I I$ chooses an arbitrary one point extension of $\sigma$.

- If $s(\sigma)=\kappa$, then $\mathcal{W}$ produces a $\beta<\delta$. Player $I I$ then chooses an $n \in \omega$ with $\delta_{n}>\beta$ and $\mathcal{W}$ produces an $\alpha$ such that $\sigma^{\frown} \alpha \in \mathcal{T}^{\dagger}$.

- If $s(\sigma)>\kappa$, then $I I$ chooses an $\eta<s(\sigma)$ and $I$ plays an $\alpha>\eta$ with $\sigma^{\frown} \alpha \in \mathcal{T}^{\dagger}$.

We note that the only places where $I$ has discretion in this game is at those $\sigma$ for which $s(\sigma)>\kappa$. Moreover, we will be interested in this game for those $\delta$ such 
that the $\delta_{n}$ are closed under the strategy $\mathcal{W}$, and hence $I I$ does not lose the game for the trivial reason of not being able to play.

\section{The winning condition:}

Player $I$ wins $\mathcal{G}_{\delta}^{2}$ iff

(1) $s k^{\mathfrak{A}}(b) \cap \kappa \subset \delta$.

(2) If $\left\langle\left(\beta_{0}, n_{0}, \alpha_{0}\right),\left(\beta_{1}, n_{1}, \alpha_{1}\right), \ldots,\left(\beta_{j}, n_{j}, \alpha_{j}\right), \ldots\right\rangle$ are the triples produced at stages $k_{j}$ where $s\left(b\left\lceil k_{j}\right)=\kappa\right.$, then $\left(\alpha_{i}, \beta_{i+1}\right) \subset\left[\delta_{n_{i}}, \delta_{n_{i}+1}\right)$.

Note. The game $\mathcal{G}_{\delta}^{2}$ is determined.

Claim 19. There is a set $D$ of measure one for the club guessing filter on $\kappa$ such that for all $\delta \in D$, player $I$ has a winning strategy in $\mathcal{G}_{\delta}^{2}$.

Proof. If not, let $A$ be a positive set of counterexamples for the club guessing filter. For each $\delta \in A$, let $\mathcal{R}_{\delta}$ be a strategy for $I I$ in $\mathcal{G}_{\delta}^{2}$.

Let $\mathfrak{A}_{1}$ be an expansion of $\mathfrak{A}$ containing the predicate for $\mathcal{T}, T^{\dagger}$, the strategy $\mathcal{W}$ for $I$ in $\mathcal{G}^{1}$ and $\left\langle\mathcal{R}_{\delta}: \delta \in A\right\rangle$.

Let $C=\left\{\gamma<\kappa: s k^{\mathfrak{A}_{1}}(\gamma) \cap \kappa=\gamma\right\}$. Let $\delta^{*} \in A$ "club guess" $C$, i.e. $\delta_{n}^{*} \in C$ for all $n$.

We claim that $I$ wins the game $G_{\delta^{*}}^{2}$. To see this we build a play of the game that obeys $I I$ 's strategy $\mathcal{R}_{\delta^{*}}$ but is a winning play for $I$.

To describe the play we only need to describe I's responses at stages $\sigma$ where $s(\sigma)>\kappa$. At these stages $\left\{\mathcal{R}_{\delta}(\sigma): \delta \in A\right.$ and $\sigma$ is a play of the game according to $\left.\mathcal{R}_{\delta}\right\}$ is bounded in $s(\sigma)$. Hence there is a "canonical" response by player $I$ against all of these strategies simultaneously. (Namely the least $\alpha$ such that $\sigma \frown \alpha \in \mathcal{T}^{\dagger}$ and $\alpha$ is a bound.) We claim that this canonical response defeats player $I I$ 's strategy $\mathcal{R}_{\delta^{*}}$.

To see this we first show that the branch $b$ produced by the play of the game $G_{\delta^{*}}^{2}$ by $I$ 's canonical strategy and the strategy $\mathcal{R}_{\delta^{*}}$ is a subset of $s k^{\mathfrak{A}_{1}}\left(\delta^{*}\right)$. Hence $s k^{\mathfrak{A}_{1}}(b) \cap \kappa \subset \delta^{*}$.

To do this we show by induction on $k$ that $b\left\lceil k \in s k^{\mathfrak{A}_{1}}\left(\delta^{*}\right)\right.$. Suppose that this is true for $k$. Let $\sigma=b\lceil k$. We have three cases:

Case 1: $s(\sigma)<\kappa$. Then, since $s(\sigma) \in s k^{\mathfrak{A}}(\sigma)$ and $\sigma \subset s k^{\mathfrak{A}_{1}}\left(\delta^{*}\right)$, we have $s(\sigma)<\delta^{*}$ and the response to $\sigma$ by player $I I$ according to $\mathcal{R}_{\delta^{*}}$ belongs to $s k^{\mathfrak{A}_{1}}\left(\delta^{*}\right)$.

Case 2: $s(\sigma)=\kappa$. In this case $I I$ chooses some $n$ and $b \uparrow(k+1)$ is determined by $\mathcal{W}$ applied to the play of the game in $\mathcal{G}^{1}$ associated with $\sigma$ and $\delta_{n}^{*}$. Since $\mathcal{W}$ and the tree $T^{\dagger}$ belong to $s k^{\mathfrak{A}_{1}}\left(\delta^{*}\right)$, we are done.

Case 3: $s(\sigma)>\kappa$. In this case the contribution by player $I$ is canonical and hence definable from $\sigma$ and $\left\langle\mathcal{R}_{\delta}: \delta \in A\right\rangle$.

In fact we need more: the definability properties are local in the following sense:

Suppose that $\left\{\left(\beta_{0}, n_{0}, \alpha_{0}\right),\left(\beta_{1}, n_{1}, \alpha_{1}\right), \ldots,\left(\beta_{k}, n_{k}, \alpha_{k}\right), \ldots\right\}$ are the plays at stages $\sigma$ with $s(\sigma)=\kappa$; then

$$
\alpha_{k}, \beta_{k+1} \in s k^{\mathfrak{A}_{1}}\left(\delta_{n_{k}+1}^{*}\right) .
$$

To see this, note that if $k_{i}$ and $k_{i+1}$ are stages in the game with $s(\sigma)=\kappa$ and no intermediate stage has label $\kappa$, then the response by $\mathcal{W}$ to produce $\beta_{i+1}$ is defined by parameters in $s k^{\mathfrak{A}_{1}}\left(\delta_{n_{i}}^{*}+1\right)$. 
To sum up we have shown that if $I$ plays her canonical strategy we get a branch $b$ through $\mathcal{T}^{\dagger}$ with the property that $s k^{\mathfrak{A}_{1}}(b) \cap \kappa \subset \delta^{*}$ and if $\left\langle\left(\beta_{0}, n_{0}, \alpha_{0}\right),\left(\beta_{1}, n_{1}, \alpha_{1}\right)\right.$, $\left.\ldots,\left(\beta_{j}, n_{j}, \alpha_{j}\right), \ldots\right\rangle$ are the triples produced at stages $k_{j}$ where $s\left(b\left\lceil k_{j}\right)=\kappa\right.$, then $\left(\alpha_{i}, \beta_{i+1}\right) \subset\left[\delta_{n_{i}}^{*}, \delta_{n_{i}+1}^{*}\right)$.

In particular there is at least one $\delta<\kappa$ such that $I$ has a winning strategy in $\mathcal{G}_{\delta}^{2}$. Fix such a $\delta$.

Repeatedly play the game using I's winning strategy to build a Namba tree $\mathcal{T}^{\prime} \subset \mathcal{T}^{\dagger}$ such that:

(1) If $\sigma \in \mathcal{T}^{\prime}$ and $s(\sigma)<\kappa$ then $\left\{\alpha: \sigma^{\frown} \alpha \in \mathcal{T}^{\prime}\right\}=\left\{\alpha: \sigma^{\frown} \alpha \in \mathcal{T}\right\}$.

(2) If $\sigma \in \mathcal{T}^{\prime}$ and $s(\sigma)>\kappa$ then $\left|\left\{\alpha: \sigma^{\frown} \alpha \in \mathcal{T}^{\prime}\right\}\right|=s(\sigma)$.

(3) At stages $\sigma$ where $s(\sigma)=\kappa$, player $I I$ plays $\left\{\delta_{n}: n \in P(\kappa)\right\}$. (The pattern at $\kappa$.

We claim that $\mathcal{T}^{\prime}$ satisfies the conclusion of Lemma [16 To see this let $b$ be a branch through $\mathcal{T}^{\prime}$. Then:

- $\sup \left(s k^{\mathfrak{A}}(b) \cap \kappa\right)=\delta$.

- If $I I$ plays $\delta_{n_{k}}$ at stage $k$, then $\alpha_{k} \in\left[\delta_{n_{k}}, \delta_{n_{k}+1}\right)$ and therefore $n_{k} \in$ $\operatorname{pat}\left(s k^{\mathfrak{A}}(b), \kappa\right)$.

- If $I I$ plays $n_{k}$ and $n_{k+1}$ at stages $\sigma, \tau$ that are successive initial segments of $b$ that satisfy $s(\sigma)=s(\tau)=\kappa$, then $\beta_{k+1} \in\left[\delta_{n_{k}}, \delta_{n_{k}+1}\right)$ and moreover,

$$
s k^{\mathfrak{A}}(b) \cap \delta_{n_{k+1}} \subset \beta_{k+1} .
$$

Note that this implies that $s k^{\mathfrak{A}}(b) \cap\left[\delta_{n_{k}+1}, \delta_{n_{k+1}}\right)=\emptyset$. In particular, there is no $n \in\left[n_{k}+1, n_{k+1}\right)$ that lies in $\operatorname{pat}\left(s k^{\mathfrak{A}}(b), \kappa\right)$.

This proves Lemma 16] and hence Theorem 15.

\section{Patterns And tightness}

4.1. Playing with patterns. In this section we discuss reflection and patterns. As remarked in the introduction (Lemma 4), if $X \prec H(\lambda)$ has uncountable cardinality and $X \cap \kappa$ has cofinality $\omega$ and pattern $r \subset \omega$, then for a closed unbounded subset $C \subset[X]^{\aleph_{0}}$, the pattern of $N \in C$ at $\kappa$ is also $r$.

This remark is our basic device for using simultaneous reflection to produce reflecting sets $X$ with uniform cofinality $\omega_{1}$. We now give corollaries of Theorem 15

Corollary 20. Suppose that for all stationary $S, T, U \subset[H(\lambda)]^{\aleph_{0}}$ there is an $X$ such that $\omega_{1}=|X| \subset X$ and $X$ reflects $S, T$ and $U$. Then for all stationary $S$ there is an $X$ that has uniform cofinality $\omega_{1}$ that reflects $S$.

Proof. Let $r, s$ be distinct infinite subsets of $\omega$. Let $T$ be the stationary set of $N \prec H(\lambda)$ such that for all regular cardinals $\kappa \leq \lambda$ with $\kappa \in N$, pat $(N, \kappa)=r$, and let $U$ be the collection of all such $N$ with $\operatorname{pat}(N, \kappa)=s$.

Then it follows from Lemma四 that any $X$ that reflects both $T$ and $U$ must have uniform cofinality $\omega_{1}$. Since $X$ reflects $S$ as well, we are done.

In particular this implies that all of the consequences of reflection to sets of uniform cofinality (such as the non-existence of good scales) follow from simultaneous reflection for any 3 stationary sets. 
We now turn to somewhat more involved uses of this device.

Corollary 21. Suppose that any pair of stationary sets simultaneously reflects to a set of size $\omega_{1}$. Let $S \subset[H(\lambda)]^{\aleph_{0}}$ be a stationary set and a be a countably infinite set of regular cardinals between $\omega_{2}$ and $\lambda$ and suppose that $\left\{\kappa_{0}, \kappa_{1}, \ldots, \kappa_{n}\right\} \subset a$. Then there is a set $X, \omega_{1}=|X| \subset X$ that reflects $S$ and there is an infinite $b \subset a$ with $\left\{\kappa_{0}, \kappa_{1}, \ldots, \kappa_{n}\right\} \subset b$ such that for all $\kappa \in b, \operatorname{cf}(X \cap \kappa)=\omega_{1}$.

Proof. By refining $S$ in $n+1$ stages we can find a stationary $S^{\prime} \subset S$ such that for all $i \leq n$, either there is an $r_{i} \subset \omega$ such that for all $N \in S^{\prime}, \operatorname{pat}\left(N, \kappa_{i}\right)=r_{i}$ or for all $r \in[\omega]^{\aleph_{0}},\left\{N \in S^{\prime}: \operatorname{pat}\left(N, \kappa_{i}\right)=r\right\}$ is non-stationary.

Fix arbitrary distinct infinite $r, s \subset \omega$ with $r \neq r_{i}$ and $s \neq r_{i}$ all $i \leq n$. Without loss of generality we can assume that for all $N \in S^{\prime}$ and all $i, \operatorname{pat}\left(N, \kappa_{i}\right) \neq r$ and $\operatorname{pat}\left(N, \kappa_{i}\right) \neq s$.

Let $T$ be the collection of $N$ such that for all $\kappa \in a, \operatorname{pat}(N, \kappa)=r$. Reflect $S^{\prime}$ and $T$ simultaneously. Then $X_{0} \cap \kappa_{i}$ must have cofinality $\omega_{1}$ for all $\left\{\kappa_{0}, \kappa_{1}, \ldots, \kappa_{n}\right\}$. If $X_{0}$ has cofinality $\omega_{1}$ relative to infinitely many $\kappa \in a$ then we are done. Otherwise we can assume that for all $N \in S \cap\left[X_{0}\right]^{\aleph_{0}}$ and all but finitely many $\kappa \in$ $a, \operatorname{pat}(N, \kappa)=r$. If this happens for a stationary collection of $X_{0}$, then by Lemma 9 there is a cofinite set $d \subset a$ such that $S^{\dagger}=\left\{N \in S^{\prime}\right.$ : for all $\left.\kappa \in d, \operatorname{pat}(N, \kappa)=r\right\}$ is a stationary set.

Let $U$ be the collection of all $N$ such that for all $\kappa \in a, \operatorname{pat}(N, \kappa)=s$. Any $X$ which reflects both $S^{\dagger}$ and $U$ must have relative cofinality $\omega_{1}$ for all but finitely many $\kappa \in a$ and also at all $\kappa \in\left\{\kappa_{0}, \kappa_{1}, \ldots, \kappa_{n}\right\}$. Since $S^{\dagger} \subset S$ we are done.

We have no immediate application of the next result; however it seems of interest:

Theorem 22. Let $S \subset[H(\theta)]^{\aleph_{0}}$ be the collection of all countable $N$ such that $\operatorname{pat}(N, \kappa) \notin N$ for all regular $\kappa \in N$ at least $\omega_{2}$. Then $S$ is stationary. In particular, $S$ has non-stationary intersection with any $S_{P}$ for $P$ a function from the regular cardinals below $\theta$ to the infinite subsets of $\omega$.

Proof. The proof is the same as in Theorem 15 except that we add a wrinkle at the very beginning. To see that $S$ is stationary we take any algebra $\mathfrak{A}=$ $\langle H(\xi), \Delta, \theta, \vec{l}, P, \ldots\rangle$ for $\xi>\theta$ and find an $N \prec \mathfrak{A}$ with $N \in S$. Note that we can assume that $\theta>2^{\aleph_{0}}$. Let $\left\langle r_{\alpha}: \alpha<\omega_{1}\right\rangle$ be an enumeration of $[\omega]^{\aleph_{0}}$ that is definable in $\mathfrak{A}$.

By an application of Lemma 14 we can assume that there is a $\delta$ such that for all branches $b$ through $\mathcal{T}_{0}, s k^{\mathfrak{A}}(b) \cap \omega_{1}=\delta$. Let $r=r_{\delta}$ and finish the proof using the real $r$ in the role of $P(\kappa)$ for all $\kappa \in s k^{\mathfrak{A}}(b)$.

4.2. Tightness. Let $\kappa$ be a singular cardinal of cofinality $\omega$ and $\left\langle\kappa_{n}: n \in \omega\right\rangle$ an increasing cofinal sequence in $\kappa$. Suppose that the true cofinality of $\prod_{n \in \omega} \kappa_{n}$ is $\kappa^{+}$ and fix a scale $\left\langle f_{\alpha}: \alpha<\kappa^{+}\right\rangle \subset \prod_{n \in \omega} \kappa_{n}$. Recall that the characteristic function of a set $A$ (relative to the $\left\langle\kappa_{n}\right\rangle$ ) is the function $\chi_{N}\left(\kappa_{n}\right)=\sup \left(N \cap \kappa_{n}\right)$. The following definition first appeared in [8].

Definition 23. Let $X \prec H(\lambda)$ have cardinality $\omega_{1}$ and $\omega_{1} \subset X$. Then $X$ is tight (with respect to $\left\langle\kappa_{n}\right\rangle$ ) iff $X \cap \prod_{n} \kappa_{n}$ is cofinal in $\prod_{n}\left(X \cap \kappa_{n}\right)$.

For countable $N \prec H(\lambda)$ we say $N$ is weakly tight iff for $\gamma_{N}=\sup \left(N \cap \kappa^{+}\right)$, $f_{\gamma_{N}} \geq^{*} \chi_{N}$. (For the notion of tightness, we refer the reader to Definition 39.) 
We will use the following characterization of tightness that appeared in $[8]$ :

Lemma 24. Let $X \prec H(\lambda)$ have cardinality $\omega_{1}$ and let $\gamma=\sup \left(X \cap \kappa^{+}\right)$. Then the following are equivalent:

- $X$ is tight and has uniform cofinality $\omega_{1}$.

- $\chi_{X}={ }^{*} f_{\gamma}$ and $\gamma$ is a good point of the scale.

The next lemma uses the ideas of the proof of the following theorem of Shelah:

Theorem (Shelah [18]). Suppose that $\kappa$ has cofinality $\omega, \lambda^{\omega}<\kappa$ for all $\lambda<\kappa$ and that $\kappa^{\omega}>\kappa^{+}$. Then there is an increasing cofinal set $\left\langle\kappa_{n}: n \in \omega\right\rangle$ in $\kappa$ and a scale $\left\langle f_{\alpha}: \alpha<\kappa^{+}\right\rangle$on $\prod \kappa_{n}$ (modulo the Frechet filter) that has the property that for all $\gamma<\kappa^{+}$with uncountable cofinality there is a closed unbounded set $D \subset \gamma$ such that

(1) o.t.D $=\operatorname{cf}(\gamma)$.

(2) For all $\beta \in D$ there is an $n<\omega$ such that $f_{\alpha}\left(\kappa_{j}\right)<f_{\beta}\left(\kappa_{j}\right)$ for all $j>n$ and all $\alpha \in D \cap \beta$.

In [4], such a sequence is called a better scale.

Lemma 25. Suppose that there is a better scale on $\prod_{n} \kappa_{n}$. Then the collection of weakly tight sets is stationary.

Proof. We assume that the sequence $\left\langle f_{\alpha}\right\rangle$ is a better scale. Let $\mathfrak{A}$ be a structure expanding $H(\lambda)$. We must produce a countable set $N \prec \mathfrak{A}$ that is weakly tight.

Let $Y \prec \mathfrak{A}$ be an internally approachable structure of cardinality $\omega_{1}$, and let $\left\langle Y_{i}: i \in \omega_{1}\right\rangle$ be a an approaching sequence. We can assume that each $Y_{i} \prec \mathfrak{A}$. Let $\gamma=\sup \left(Y \cap \kappa^{+}\right)$and $\gamma_{i}=\sup \left(Y_{i} \cap \kappa^{+}\right)$. Then $\left\{\gamma_{i}: i \in \omega_{1}\right\}$ is a closed unbounded set in $\gamma$. Let $D \subset \gamma$ be the closed unbounded set guaranteed to exist from the definition of "better scale". Then there is a stationary subset $S$ of $D \cap\left\{\gamma_{i}\right\}_{i<\omega_{1}}$ and a $k \in \omega$ such that for all $n>k, \gamma_{i}<\gamma_{j} \in S, f_{\gamma_{i}}\left(\kappa_{n}\right)<f_{\gamma_{j}}\left(\kappa_{n}\right)$. For each $\gamma_{i} \in S$, there is a $k_{i}$ such that $\chi_{Y_{i}}\left(\kappa_{n}\right)<f_{\gamma_{j}}\left(\kappa_{n}\right)$ for all $n>k_{i}$ and all $\gamma_{j} \in S \backslash\left\{\gamma_{i}+1\right\}$. By refining $S$ again we can assume that for all $\gamma_{i} \in S, k_{i}=k$.

Let $\gamma^{*}={ }_{\text {def }} \gamma_{i^{*}} \in S$ be a limit point of $S$. We claim that $Y^{*}={ }_{\text {def }} Y_{i^{*}}$ is weakly tight. To see this we note that:

$$
\chi_{Y^{*}}=\sup _{i<i^{*}} \chi_{Y_{i}}
$$

pointwise, and for all $n>k$,

$$
\sup _{i<i^{*}} f_{\gamma_{i}}\left(\kappa_{n}\right) \leq f_{\gamma^{*}}\left(\kappa_{n}\right) \text {. }
$$

On the other hand for $\gamma_{i}<\gamma_{j}<\gamma_{l} \in S \cap \gamma^{*}$ and $n>k$,

$$
\chi_{Y_{i}}\left(\kappa_{n}\right)<f_{\gamma_{j}}\left(\kappa_{n}\right)<\chi_{Y_{l}}\left(\kappa_{n}\right) .
$$

So for all $n>k, \sup _{i<i^{*}} \chi_{Y_{i}}\left(\kappa_{n}\right)=\sup _{i<i^{*}} f_{\gamma_{i}}\left(\kappa_{n}\right)$ and we are done.

The next observation will be used in the proof of the singular cardinals hypothesis.

Lemma 26. Let $X$ be a set of cardinality $\omega_{1}$ and suppose that cf $\left(X \cap \kappa^{+}\right)$has cofinality $\omega_{1}$. If $X$ reflects the collection of weakly tight structures, then:

(1) $X$ is tight.

(2) $X \cap \kappa_{n}$ has cofinality $\omega_{1}$ for almost all $n$. 
Proof. Let $a \subset X$ be countable. It suffices to show that there is a function $f \in$ $X \cap \prod_{n} \kappa_{n}$ such that for all but finitely many $n, f\left(\kappa_{n}\right) \geq \sup \left(a \cap \kappa_{n}\right)$. Since the collection of weakly tight sets is stationary in $X$, there is a weakly tight $N \subset X$ with $a \subset N$. Then $f_{\gamma_{N}}\left(\kappa_{n}\right) \geq \sup \left(a \cap \kappa_{n}\right)$ for all but finitely many $N$. Since $X \cap \kappa^{+}$ has cofinality $\omega_{1}$, there is a $\gamma \in X$ such that $\gamma>\gamma_{N}$. Thus $f_{\gamma}\left(\kappa_{n}\right) \geq \sup \left(a \cap \kappa_{n}\right)$ for almost all $n$.

\section{ThE SINGULAR CARDinAls HYPOTHESIS}

In this section we describe a set that must be closed unbounded if every pair of stationary sets simultaneously reflects. On the other hand we will show that it is co-stationary if the singular cardinals hypothesis fails.

Definition 27. Let $\kappa$ be a singular cardinal of cofinality $\omega$ and $\left\langle\kappa_{n}: n \in \omega\right\rangle$ a cofinal sequence of regular cardinals. A pre-Jensen matrix is a matrix of sets $\left\langle A_{\alpha}^{n}: n \in \omega, \alpha<\kappa^{+}\right\rangle$satisfying:

(1) For all $\alpha, n, A_{\alpha}^{n} \subset \kappa^{+}$and $\left|A_{\alpha}^{n}\right|=\kappa_{n}$.

(2) For all $\alpha<\beta, n<\omega$ there is an $m<\omega, A_{\alpha}^{n} \subset A_{\beta}^{m}$.

(3) $\bigcup_{n \in \omega} A_{\alpha}^{n}=\alpha$.

(For the record a Jensen Matrix has the additional properties that for all $\alpha$ having uncountable cofinality,

$$
\bigcup_{n \in \omega}\left[A_{\alpha}^{n}\right]^{\aleph_{0}}=\bigcup_{n \in \omega, \beta<\alpha}\left[A_{\beta}^{n}\right]^{\aleph_{0}}
$$

and that $\bigcup_{n \in \omega, \beta<\kappa^{+}}\left[A_{\beta}^{n}\right]^{\aleph_{0}}=\left[\kappa^{+}\right]^{\aleph_{0}}$. For more information about Jensen matrices and related square principles the reader is referred to [10].)

Lemma 28. There is a pre-Jensen matrix.

Proof. Let $\lambda$ be a regular cardinal much bigger than $\kappa$ and let $\mathfrak{A}=\langle H(\lambda), \in, \Delta, \ldots\rangle$ be as usual. We define our sequence by induction on $\alpha$. Suppose that we have defined $A_{\alpha}^{n}$ for all $\alpha<\beta$.

We will define a sequence $\left\langle M_{k}: k \in \omega\right\rangle$ and let $A_{\beta}^{m}=M_{\infty} \cap \kappa^{+}$where $M_{\infty}=$ $\bigcup_{k \in \omega} M_{k}$. Choose a cofinal sequence $C \subset \beta$. Let $M_{0}=s k^{\mathfrak{A}}\left(\kappa_{m} \cup C\right)$. Let $M_{i+1}$ be the Skolem hull in $\mathfrak{A}$ of

$$
M_{i} \cup \bigcup_{\beta \in M_{i} \cap \alpha, n<m} A_{\beta}^{n} .
$$

Then $M_{\infty}$ has cardinality $\kappa_{m}$ and if $\alpha \in A_{\beta}^{m}$, then $A_{\alpha}^{n} \subset A_{\beta}^{m}$ for all $n<m$. Finally it is easy to check that if $n<m$, then $A_{\beta}^{n} \subset A_{\beta}^{m}$, and that $\bigcup_{m \in \omega} A_{\beta}^{m}=$ $\beta$.

Definition 29. Suppose that the true cofinality of $\prod_{n \in \omega} \kappa_{n}$ is $\kappa^{+}$and fix a scale $\left\langle f_{\alpha}: \alpha<\kappa^{+}\right\rangle \subset \prod_{n \in \omega} \kappa_{n}$.

- For a countable set $A \subset \kappa^{+}$, we define $\Gamma_{A}\left(\kappa_{n}\right)=\sup _{\alpha \in A}\left(f_{\alpha}\left(\kappa_{n}\right)\right)$ for all $n \in \omega$. (We remark that for $N \prec H(\lambda), \Gamma_{N \cap \kappa^{+}}=\chi_{N}$.)

- Let $\mathcal{F}$ be the subset of $\prod_{n} \kappa_{n}$ defined to be the collection of all $g \in \prod_{n} \kappa_{n}$ such that for some $\alpha<\kappa^{+}, n \in \omega$ and $X \in\left[A_{\alpha}^{n}\right]^{\aleph_{0}}$ for all but finitely many $k, g\left(\kappa_{k}\right)=\Gamma_{X}\left(\kappa_{k}\right)$.

- Define $\mathcal{S}$ to be the collection of countable $A \subset \kappa^{+}$such that $\Gamma_{A} \notin \mathcal{F}$. 
Lemma 30. Suppose that $\left\langle f_{\alpha}: \alpha<\kappa^{+}\right\rangle$is a good scale. Then for a closed unbounded set of $X \in[H(\lambda)]^{\aleph_{1}}$ if $c f\left(X \cap \kappa^{+}\right)$is $\omega_{1}$ and $X$ is tight, then $X$ does not reflect $\mathcal{S}$.

Proof. Suppose $X \prec\left\langle H(\lambda), \in, \Delta,\left\langle f_{\alpha}\right\rangle_{\alpha}, \ldots\right\rangle$ satisfies the hypothesis of the lemma.

Let $\gamma=\sup \left(X \cap \kappa^{+}\right)$and $a=\left\{\kappa_{n}\right\}$. Since $\left\langle f_{\alpha}\right\rangle$ is a good scale, we can find a cofinal set $B \subset X \cap \gamma$ such that for some finite $b \subset a$ and all $\kappa \in a \backslash b,\left\{f_{\alpha}(\kappa): \alpha \in B\right\}$ is a strictly increasing sequence of length $\omega_{1}$ and $\chi_{X}(\kappa)=\sup \left\{f_{\alpha}(\kappa): \alpha \in B\right\}$. Since $B$ is uncountable, there is an $n,\left|B \cap A_{\gamma}^{n}\right|=\omega_{1}$, and hence we can assume that $B \subset A_{\gamma}^{n}$.

Note that there is a closed unbounded collection of countable subsets $N$ of $X$ such that for all $\alpha \in N$ there is a $\delta \in B \cap N$ such that for all $\kappa \in a \backslash b, f_{\alpha}(\kappa)<f_{\delta}(\kappa)$.

However this implies that the collection of countable subsets $N$ of $X$ for which $\Gamma_{N}(\kappa)=\sup _{\delta \in B^{\prime}} f_{\delta}(\kappa)$ for some countable $B^{\prime} \subset B$ and all but finitely many $\kappa \in a$ contains a closed unbounded set. Hence the collection of $N$ such that $\Gamma_{N} \in \mathcal{F}$ is closed unbounded in $[X]^{\aleph_{0}}$ and hence $\mathcal{S}$ cannot reflect to $X$.

We now establish the singular cardinals hypothesis.

Theorem 31. Suppose that for all $\lambda$ every pair of stationary sets in $[H(\lambda)]^{\aleph_{0}}$ simultaneously reflects to a stationary set of size $\omega_{1}$. Then for all regular cardinals $\kappa \geq \omega_{2}$ :

$\kappa^{\omega}=\kappa$.
(In particular, for all $\kappa$ of cofinality $\omega, \kappa^{\omega}=\kappa^{+}$.)

By 22, if every stationary set reflects to a set of size $\omega_{1}$, then $2^{\aleph_{0}} \leq \omega_{2}$. Hence, the second assertion is equivalent to the first and this is what we establish. (See [20] or [11].)

The basic outline of the proof is as follows. Suppose that the set $\mathcal{S}$ given in Definition 29 is stationary. To obtain a contradiction we work to reflect $\mathcal{S}$ to a tight set $X$, which is impossible by Lemma 30. To reflect $\mathcal{S}$ to a tight set, we simultaneously reflect $\mathcal{S}$ with the stationary set of weakly tight structures (which form a stationary set since the SCH fails at $\kappa$ (Lemma 25)). The two problematic points are seeing that $\mathcal{S}$ is stationary and that when reflected simultaneously with the weakly tight sets, the result is an $X$ that has cofinality $\omega_{1}$ at $\kappa^{+}$. To achieve the latter we again resort to the device of "patterns".

Definition 32. Suppose that $r \subset \omega$ is infinite. Define $\mathcal{S}_{r} \subset \mathcal{S}$ to be the collection of $N \in \mathcal{S}$ such that $\operatorname{pat}\left(N, \kappa^{+}\right)=r$.

Lemma 33. If $\kappa^{\omega}>\kappa^{+}$and $r \subset \omega$ is infinite, then the set $\mathcal{S}_{r}$ is stationary.

Remark. Assuming this lemma we can carry out the outline described above. We first fix any infinite $r \subset \omega$; then (by Lemmas 26 and 301) the only $X \in[H(\lambda)]^{\aleph_{1}}$ that simultaneously reflect both $\mathcal{S}_{r}$ and the weakly tight structures are those with $\operatorname{cof}\left(X \cap \kappa^{+}\right)=\omega$. Hence $T_{r}=\operatorname{def}\left\{N: N\right.$ is weakly tight and $\left.\operatorname{pat}\left(N, \kappa^{+}\right)=r\right\}$ is stationary. Now take any infinite $s \subset \omega$ that differs from $r$ and reflect $T_{r}$ simultaneously with $\mathcal{S}_{s}$. The result is a tight set $X \in[H(\lambda)]^{\aleph_{1}}$ that has $\operatorname{cof}\left(X \cap \kappa^{+}\right)=\omega_{1}$ and reflects $\mathcal{S}_{s}$ (and hence $\mathcal{S}$ ). This contradiction finishes the proof of Theorem 31 We note that were we content to prove the SCH from reflecting any three sets simultaneously, the argument would simplify to showing that $\mathcal{S}$ is stationary. Thus it suffices to show Lemma 33 
Proof. We show that for any structure $\mathfrak{A}$ expanding some large $\langle H(\lambda), \in, \Delta\rangle$ there is a $\delta<\kappa^{+}$and family $\mathcal{D}$ of elementary substructures of $\mathfrak{A}$ with supremum $\delta$ and pattern $r$ such that $\left\{\chi_{N}: N \in \mathcal{D}\right\}$ has cardinality $\kappa^{\omega}$. This suffices by a simple cardinality argument, since the cardinality of $\mathcal{F}$ is $\kappa^{+}$. To do this we recapitulate portions of the proof of Theorem 15 with suitable modifications.

In our current situation we need only play one game, as we are not concerned about cardinals above $\kappa^{+}$. We play the game on Namba subtrees of the tree $\mathcal{T}=$ $\left\{\sigma \in\left[\kappa^{+}\right]^{<\omega}: \sigma(2 n) \in \kappa_{n}\right.$ and $\left.\sigma(2 n+1) \in \kappa^{+} \cap \operatorname{cof}\left(\omega_{1}\right)\right\}$. In the game $\mathcal{G}$ on some subtree $\mathcal{T}^{*} \subset \mathcal{T}$ players $I$ and $I I$ play according to the following schedule. We assume that we want to extend a partial play $\sigma \in \mathcal{T}^{*}$ :

(1) At an even stage $2 n$, player $I I$ plays an ordinal $t_{n}<\kappa_{n}$ with $\sigma^{\frown} t_{n} \in \mathcal{T}^{*}$.

(2) At odd stages $2 n+1$, player $I$ plays an ordinal $\beta_{n}<\kappa^{+}$, II plays an ordinal $\gamma_{n}>\beta_{n}$ and $I$ plays an $\alpha_{n}>\gamma_{n}$ with $\sigma^{\frown} \alpha_{n} \in \mathcal{T}^{*}$.

Player $I$ wins $\mathcal{G}$ iff

$$
s k^{\mathfrak{A}}\left(\left\{\left\{t_{n}, \alpha_{n}\right\}: n \in \omega\right\}\right) \cap \bigcup_{n}\left[\beta_{n}, \gamma_{n}\right)=\emptyset .
$$

Claim 34. There is a subtree $\mathcal{T}^{*} \subset \mathcal{T}$ such that player $I$ has a winning strategy on the game played on $\mathcal{T}^{*}$ and $\mathcal{T}^{*}$ is Namba in the sense that:

(1) For $\sigma \in \mathcal{T}^{*}$ of even length $2 n,\left|\left\{t: \sigma \frown t \in \mathcal{T}^{*}\right\}\right|=\kappa_{n}$.

(2) For $\sigma \in \mathcal{T}^{*}$ of odd length, $\left|\left\{\alpha: \sigma^{\frown} \alpha \in T^{*}\right\}\right|=\kappa^{+}$(the successors of $\sigma$ are even stationary; see below).

For each $\alpha \in \kappa \cap \operatorname{cof}\left(\omega_{1}\right)$, we choose $\left\langle\alpha(i): i \in \omega_{1}\right\rangle$ converging to $\alpha$. For each infinite branch $c$ through $\mathcal{T}$ and each $\alpha_{n} \in c$ from a $\kappa^{+}$-splitting node, let $i_{n}^{c}$ be the least $i \in \omega_{1}$ such that $\alpha_{n}(i)>s k^{\mathfrak{A}}(c) \cap \alpha$. Let $i(c)=\sup \left\langle i_{n}^{c}: n \in \omega\right\rangle$.

Arguing exactly as in Theorem 15 (and Claim 17) we see that we can build a tree $\mathcal{T}^{*} \subset \mathcal{T}$ that has the following properties:

(1) If $\tau$ has odd length $2 n+1$, then $\left|\left\{t: \tau^{\frown} t \in \mathcal{T}^{*}\right\}\right|=\kappa_{n}$.

(2) if $\tau$ has even length, then $\left\{\alpha<\kappa^{+}: \tau \frown \alpha \in \mathcal{T}\right\}$ is stationary.

(3) There is an ordinal $\beta^{*}$ such that for all branches $c$ through $\mathcal{T}^{*}, i(c)=\beta^{*}$.

We claim that $I$ has a winning strategy on this tree. As before we define a strategy for player $I$. Player $I$ only has discretion to play at odd stages. Here he considers the sequence $\left(t_{0}, \alpha_{0}, \ldots, t_{n}\right)$ already played. Since the set $O=\{\alpha$ : $\left.\left(t_{0}, \alpha_{0}, \ldots, t_{n}, \alpha\right) \in \mathcal{T}^{*}\right\}$ is stationary, he can find a $\beta_{n}$ such that for a stationary set $G \subset O$ if $\alpha \in G$ then $\alpha\left(\beta^{*}\right)=\beta_{n}$. He plays this $\beta_{n}$. When player $I I$ responds with $\gamma_{n}$, player $I$ plays some $\alpha_{n} \in G$ greater than $\gamma_{n}$.

By item (3), if $\left\{t_{n}, \alpha_{n}: n \in \omega\right\}$ is a play produced this way, then we see that $s k^{\mathfrak{A}}\left(\left\{t_{n}, \alpha_{n}\right\}_{n}\right) \cap \alpha_{n} \subset \beta_{n}$. In particular

$$
s k^{\mathfrak{A}}\left(\left\{t_{n}, \alpha_{n}\right\}_{n}\right) \cap \bigcup_{n}\left[\beta_{n}, \gamma_{n}\right)=\emptyset .
$$

Hence this play is a win for player $I$; thus we have defined a winning strategy for the first player in the game. This finishes the proof of Claim 34.

Let $\mathcal{W}$ be a winning strategy for $I$ in the game. Let $D$ be the closed unbounded set of $\delta$ such that $s k^{\mathfrak{A}}(\delta \cup\{\mathcal{W}\}) \cap \kappa^{+}=\delta$. Choose a $\delta$ where the ladder system for $\kappa^{+}$is defined that is such that $\left\langle\delta_{n}: n \in \omega\right\rangle \subset D$. In particular, note that if $\sigma$ is a partial play of the game $\mathcal{G}$ according to $\mathcal{W}$, then any $\gamma<\delta_{n}$ played at an odd stage in the game, has a response via $\mathcal{W}$ of an $\alpha<\delta_{n}$. 
We now build a large collection of structures with pattern $r \subset \omega$ that have supremum $\delta$. Enumerate $r$ in increasing order as $\left\langle k_{l}: l \in \omega\right\rangle$. As in Claim [18, we can build a tree $\mathcal{T}^{\dagger} \subset \mathcal{T}$ such that:

(1) Every branch $b$ through $\mathcal{T}^{\dagger}$ is a sequence of ordinals $\left\langle t_{0}, \alpha_{0}, t_{1}, \alpha_{1}, \ldots\right\rangle$ that is the result of a play of the game $\mathcal{G}$ according to $\mathcal{W}$.

(2) For $\tau \in \mathcal{T}^{\dagger}$ of odd length, $\left|\left\{t: \tau \frown t \in \mathcal{T}^{\dagger}\right\}\right|=\kappa_{n}$.

(3) If $\sigma \in \mathcal{T}^{\dagger}$ has length $2 l+1$, then $\alpha_{l}$ is the result of $I I$ playing the ordinal $\delta_{n}$ where $n=k_{l}$.

To see that item (3) is possible, we note that $\delta_{n}$ is closed under the strategy $\mathcal{W}$. Since (inductively) $\sigma \subset \delta_{n}$, any $\beta_{l}$ played according to $\mathcal{W}$ is less than $\delta_{n}$.

Now suppose that $b$ is a branch through $\mathcal{T}^{\dagger}$. We claim that $s k^{\mathfrak{A}}(b)$ has pattern $r$. Let $\left\{t_{l},\left(\beta_{l}, \gamma_{l}, \alpha_{l}\right): l \in \omega\right\}$ be the ordinals played during the game. If $n=k_{l} \in r$ then at stage $2 l+1$ player $I I$ played $\delta_{n}\left(\right.$ as $\left.\gamma_{l}\right)$. Since $\delta_{n+1} \in D, \alpha_{l}<\delta_{n+1}$. Hence $n \in \operatorname{pat}\left(S k^{\mathfrak{A}}(b), \kappa\right)$. Hence $r \subset \operatorname{pat}\left(S k^{\mathfrak{A}}(b), \kappa\right)$. On the other hand if $n \in\left(k_{l}, k_{l+1}\right)$ (where $k_{l}$ and $k_{l+1}$ are successive elements of $r$ ), then at stage $2 l+1$, player $I I$ played $\delta_{k_{l}}$. Since $\delta_{k_{l}+1} \in D, \beta_{l+1}<\delta_{k_{l}+1}$. At stage $2 l+3$ player $I I$ played $\delta_{k_{l+1}}$ as $\gamma_{l+1}$. Since $\mathcal{S}$ is a winning strategy, $s k^{\mathfrak{A}}(b) \cap\left[\beta_{l+1}, \delta_{k_{l+1}}\right)=\emptyset$. In particular, $n \notin \operatorname{pat}\left(s k^{\mathfrak{A}}(b), \kappa\right)$.

We have a continuous, one to one map from $\prod_{n} \kappa_{n}$ into branches through $\mathcal{T}^{\dagger}$. Thus we can associate (in a continuous way) to each $f \in \prod_{n} \kappa_{n}$ an $N_{f} \prec \mathfrak{A}$ with $\sup \left(N_{f} \cap \kappa^{+}\right)=\delta$ and $\operatorname{pat}\left(N_{f}, \kappa\right)=r$.

Applying Theorem[14 repeatedly, we inductively construct a tree $\mathcal{R} \subset \kappa^{<\omega}$ such that:

(1) If $\sigma \in \mathcal{R}$ has length $n$, then $\left\{\alpha: \sigma^{\frown} \alpha \in \mathcal{R}\right\} \subset \kappa_{n}$ and has cardinality $\kappa_{n}$.

(2) If $f, g$ are infinite branches through $\mathcal{R}$ and $f(n)<g(n)$, then $\sup \left(N_{f} \cap \kappa_{n}\right)<$ $\sup \left(N_{g} \cap \kappa_{n}\right)$.

In particular, any two distinct branches $f, g$ through $\mathcal{R}$ yield distinct $N_{f}, N_{g}$. This proves Lemma 33 and hence Theorem 31

\section{Some REMARKS On RECONSTRUCTION}

In this section we discuss a technique for reconstructing a countable set from a finite set of ordinals. We use this to show that there is a particular stationary set of countable structures that, if reflected to a set $X$ of cardinality $\omega_{1}$, has stationary intersection with $P_{\omega_{1}}(X) \cap X$.

We will use some square principles whose ZFC status is currently unknown.

Definition 35. Let $\kappa$ be a cardinal. A partial square sequence for $\kappa$ is a sequence $\mathcal{C}=\left\langle C_{\alpha}: \alpha \in S\right\rangle$ of sets such that:

- $C_{\alpha} \subset \alpha$ is closed and unbounded.

- o.t. $\left(C_{\alpha}\right)<\kappa$.

- If $\gamma$ is a limit point of both $C_{\alpha}$ and $C_{\beta}$, then $C_{\alpha} \cap \gamma=C_{\beta} \cap \gamma$.

If $S$ is a stationary set of a fixed cofinality $\mu$, then we will also assume that the order type of each $C_{\alpha}$ is $\mu$.

Note that if $\mathcal{C}$ is a partial square sequence, then it can be extended to a partial square sequence on $\left\{\gamma\right.$ : for some $\alpha, \gamma$ is a limit point of $\left.C_{\alpha}\right\}$. 
The following conjecture has been shown by Shelah [18] for $\kappa$ a successor of a regular cardinal at least $\aleph_{3}$ :

Conjecture. If $\kappa$ is a successor cardinal, then there is a stationary set $S \subset$ $\operatorname{cof}\left(\omega_{1}\right) \cap \kappa$ on which there is a partial square sequence.

We will assume the conjecture for the rest of this section. For most of our results the conjecture can be loosened to require only that $S$ be a stationary subset of $\operatorname{cof}\left(\omega_{n}\right)$ for some $n$; however we do not give the arguments here.

We will make the following assumption on the rationalization that it is a consequence of multiple reflection:

Assumption. For all singular $\kappa,|p c f(\kappa)|=1$.

We illustrate the ideas at $\aleph_{\omega+1}$; the ideas carry over very similarly to general $\kappa$ below the first fixed point of the $\aleph$ function. (Where our knowledge of the PCF theory ends.) Because we are working aroung $\aleph_{\omega}$, in this section the characteristic functions are relative to $\prod_{n \geq 2} \aleph_{n}$, and if $f \in \prod \aleph_{n}$, we will often write $f(n)$ instead of $f\left(\omega_{n}\right)$.

We start with a well-known lemma that is the basis for our reconstruction technique.

Lemma 36. Suppose that $N, M$ are elementary substructures of the structure $\mathfrak{A}_{0}=\left\langle H\left(\aleph_{\omega+1}\right), \in, \Delta\right\rangle$ and are such that for all $2 \leq n \in \omega, \chi_{N}(n) \leq \chi_{M}(n)$, $\sup \left(N \cap \omega_{1}\right) \leq \sup \left(M \cap \omega_{1}\right)$ and for all $n \leq \omega, N \cap M$ is cofinal in $N \cap \aleph_{n+1}$. Then $N \cap \aleph_{\omega+1} \subset M \cap \aleph_{\omega+1}$.

Suppose that $S \subset \aleph_{\omega+1}$ is a stationary set and $\mathcal{C}=\left\langle C_{\alpha}: \alpha \in S\right\rangle$ is a partial square sequence on $S$. Extend $\mathcal{C}$ to a partial square sequence on the collection of $\gamma$ that are limit points of some $C_{\alpha}$.

Fix a scale $\left\langle f_{\alpha}: \alpha<\aleph_{\omega+1}\right\rangle \subset \prod_{n \geq 2} \aleph_{n}$.

Lemma 37. There is a sequence $\left\langle f_{\alpha}^{\prime}: \alpha<\aleph_{\omega+1}\right\rangle$ and $a k \in \omega$ such that each $f_{\alpha}^{\prime}={ }_{k} f_{\alpha}$ and is such that for each club set $D,\left\{f_{\alpha}^{\prime}: \alpha \in D \cap S\right\}$ dominates every function in $\prod_{n \geq 2} \aleph_{n}$ everywhere.

Proof. Split $S$ into $\aleph_{\omega+1}$ disjoint stationary sets $\left\{S_{i}: i \in \aleph_{\omega+1}\right\}$. We claim that for each $i$ there is a $k_{i}$ such that for all closed unbounded sets $D,\left\{f_{\alpha}: \alpha \in\right.$ $\left.S_{i} \cap D\right\}$ dominates $\prod_{n>k_{i}} \omega_{n}$ everywhere. For otherwise for each $k$ we would have a counterexample $g_{k}, D_{k}$ with the property that for all $\alpha \in S_{i} \cap D_{k}$, there is an $n>$ $k, f_{\alpha}(n) \leq g_{k}(n)$. Letting $g$ be the pointwise supremum of the $g_{k}$ and $D=\bigcap_{k} D_{k}$ we see that for no $\alpha \in S_{i} \cap D$ is $g<^{*} f_{\alpha}$, a contradiction.

Hence for some $k$ there are $\aleph_{\omega+1}$ many $S_{i}$ 's with $k_{i}=k$. Choose a one to one map that associates to each $\vec{\gamma} \in \prod_{1 \leq n \leq k} \aleph_{n}$ an $i=i_{\vec{\gamma}}$ with $k_{i}=k$, and for $\alpha \in S_{i_{\vec{\gamma}}}$ define

$$
f_{\alpha}^{\prime}(n)= \begin{cases}\vec{\gamma}(n) & \text { if } n \leq k, \\ f_{\alpha}(n) & \text { otherwise }\end{cases}
$$

Let $D$ be any closed unbounded set and $g \in \prod_{n \geq 2} \aleph_{n}$. Let $\vec{\gamma} \in \prod_{1<n \leq k} \aleph_{n}$ be everywhere larger than $g \uparrow(k+1)$. Then there is some $\alpha \in S_{i_{\vec{\gamma}}} \cap D$ such that $g<_{k} f_{\alpha}$. But then $g$ is everywhere less than $f_{\alpha}^{*}$, as desired.

We remind the reader (as in the remarks after Lemma 25) that if the SCH fails at $\kappa \in \operatorname{cof}(\omega)$, then there is a cofinal set $\left\langle\kappa_{n}: n \in \omega\right\rangle$ in $\kappa$ with a scale of length $\kappa^{+}$in $\prod \kappa_{n}$. 
Definition 38. We say that the scale has been squared iff for all $\gamma$ in the domain of $\mathcal{C}$ and all $n$ such that $\omega_{n}>c f(\gamma), f_{\gamma}(n)=\sup \left\{f_{\delta}(n): \delta \in C_{\gamma}\right\}$.

Note that any scale can be squared by induction and that squaring a scale twice is the same as squaring a scale once. Moreover, if $\gamma$ is an ordinal where the square sequence has been defined, then for all $n$ the function sending $\delta$, a limit point of $C_{\gamma}$, to $f_{\delta}(n)$ is strictly increasing.

For each $2 \leq n \in \omega$, fix a ladder system $\vec{l}\left(\omega_{n}\right)$ on a stationary set $S_{n} \subset \omega_{n} \cap \operatorname{cof}(\omega)$ that associates to each $\delta \in S_{n}$ an increasing sequence $\left\langle\delta_{n}: n \in \omega\right\rangle$.

Definition 39. Let $N \prec \mathfrak{A}_{0}$ be countable. Recall that $N$ is weakly tight iff $\chi_{N} \leq^{*}$ $f_{\gamma}$ where $\gamma=\sup \left(N \cap \aleph_{\omega+1}\right)$.

$N$ is tight iff $N$ is weakly tight, $C_{\gamma}$ exists and for some $k$, for all $n \geq k,\left\{f_{\delta}(n)\right.$ : $\delta$ is a limit point of $\left.C_{\gamma}\right\} \subset N \cap \aleph_{n}$. (In particular tightness implies that $\chi_{N}={ }_{k} f_{\gamma}$.)

$N$ is reconstructible iff $N$ is tight, $\chi_{N}={ }_{k} f_{\gamma}$ and for all $1<j<k$ the club guessing sequence is defined at $\delta=\sup \left(N \cap \omega_{j}\right)$ and $\left\langle\delta_{n}: n \in \omega\right\rangle \subset N$.

We note that if $\chi_{N} \leq^{*} f_{\gamma}$ and $C_{\gamma} \subset N$ where $\gamma=\sup \left(N \cap \aleph_{\omega+1}\right)$, then $N$ is tight. In practice this is the form we shall use.

Lemma 40. Let $\left\langle f_{\alpha}: \alpha\left\langle\aleph_{\omega+1}\right\rangle\right.$ be a scale that has been squared by a partial square sequence $\mathcal{C}$ defined on a stationary set $S \subset \aleph_{\omega+1} \cap \operatorname{cof}\left(\omega_{1}\right)$. The collection of reconstructible structures is stationary.

Proof. Fix an algebra $\mathfrak{A}$ on $H(\theta)$ extending $\mathfrak{A}_{0}$. Without loss of generality we can assume that $\mathfrak{A}$ contains a predicate for the partial square sequence $\mathcal{C}$ and for the club guessing ladder systems. Choose an algebra $\mathfrak{B}$ on $H(\theta)$ containing the function $X \mapsto s k^{\mathfrak{A}}(X)$ for $X \in H(\theta)$. Let $\gamma \in S$ be an ordinal of cofinality $\omega_{1}$ such that $s k^{\mathfrak{B}}(\gamma) \cap \aleph_{\omega+1}=\gamma$.

Define a sequence of ordinals $\left\langle\gamma_{i}: i \in \omega_{1}\right\rangle$ by induction with the property that for all $i>0, \gamma_{i} \in C_{\gamma}$. Let $\gamma_{0}=0$. Suppose that $\left\langle\gamma_{j}: j<i\right\rangle$ is defined. Let $N_{i}=s k^{\mathfrak{A}}\left(\left\langle\gamma_{j}: j<i\right\rangle\right)$. Let $\gamma_{i}$ be the least ordinal $\eta$ in the limit points of $C_{\gamma}$ such that $\chi_{N_{i}}<* f_{\eta}$.

For each $i$ there is a $k=k_{i}$ such that $\chi_{N_{i}}<_{k} f_{\gamma_{i}}$. So stationarily many $i$ have the same $k_{i}$. Let $A$ be an infinite subset of $\omega_{1}$ such that for some $k$ and all $i \in A, k_{i}=k$. Without loss of generality we can take $A$ to have order type $\omega$. Let $\delta=\sup \left\{\gamma_{i}: i \in A\right\}$. Then $\delta \in C_{\gamma}$.

We claim that if $N=s k^{\mathfrak{A}}\left(\left\{\gamma_{i}: i \in A\right\}\right)$ then $\chi_{N} \leq_{k} f_{\delta}$. To see this, note that $N \subset \bigcup_{i \in A} N_{i}$, and so $\chi_{N}(n) \leq \sup _{i \in A} \chi_{N_{i}}(n)$. But each $\chi_{N_{i}}<_{k} f_{\gamma_{i}}$ and $f_{\delta}$ is the pointwise supremum of the $f_{\gamma_{i}}$ since the scale has been squared.

Since $\delta$ is a limit point of the $\gamma_{i} \in A$ and each $\gamma_{i}$ is a limit point of $C_{\gamma}$, and $\mathfrak{A}$ has a predicate for $\mathcal{C}$, we see that each $C_{\gamma_{i}} \subset N$. Hence $C_{\delta} \subset N$ and $N$ is tight.

Let $N_{0}=s k^{\mathfrak{A}}\left(N \cup \omega_{k}\right)$. By Lemma 10, $\chi_{N_{0}}={ }_{k} \chi_{N}$ and $\sup \left(N_{0} \cap \aleph_{\omega+1}\right)=\delta$. We now show by induction on $1 \leq m \leq k$ that there is an $N_{m} \prec N_{0}$ with the properties that:

(1) $\left|N_{m}\right|=\omega_{k-m} \subset N_{m}$.

(2) $\sup \left(N_{m} \cap \aleph_{\omega+1}\right)=\delta$ and $C_{\delta} \subset N_{m}$.

(3) $\chi_{N_{m}}={ }_{k} \chi_{N}$.

(4) If $\delta(m)=\sup \left(N_{m} \cap \omega_{k-m+1}\right)$, then for all $1 \leq m \leq k-1, \delta(m) \in S_{k-m+1}$.

(5) For all $1 \leq m<k$, and $m \leq m^{\prime},\left\langle\delta(m)_{n}: n \in \omega\right\rangle \subset N_{m^{\prime}}$. 
Suppose that we have defined $N_{m-1}$ and $m<k$. Let $M_{m-1}$ be the structure expanding $N_{m-1}$ by adding predicates for $C_{\delta}$, and $\left\langle\delta(l)_{n}: n \in \omega, 1 \leq l<m\right\rangle$.

Let $D=\left\{\gamma<\omega_{k-m}: s k^{M_{m-1}}(\gamma) \cap \omega_{k-m+1}=\gamma\right\}$. Then $D$ is a closed unbounded set and so there is a $\delta(m) \in S_{m}$. In particular, $s k^{M_{m-1}}\left(\left\{\delta(m)_{n}: n \in \omega\right\}\right) \cap$ $\omega_{k-m+1}=\delta(m)$. Letting $N_{m}=s k^{M_{m-1}}\left(\left\{\delta(m)_{n}: n \in \omega\right\} \cup \omega_{k-m}\right)$ we finish the induction for $m<k$.

Given $N_{k-1}$ we define $N_{k}$ to be any countable elementary substructure of $N_{k-1}$ containing the sets required by items (2) and (5). We check that $N_{k}$ works. Clearly $N_{k} \prec \mathfrak{A}$ and $C_{\delta} \subset N_{k} \subset N_{0}$, and hence $N_{k}$ is tight. Moreover, $\chi_{N_{k}}={ }_{k} f_{\delta}$, for $1<m<k,\left\langle\delta(k-m)_{n}: n \in \omega\right\rangle \subset N_{k}$.

We have shown that given any structure $\mathfrak{A}$ we can find an elementary substructure that is reconstructible. The lemma follows.

Theorem 41. Let $\lambda$ be a large regular cardinal. Suppose that there is a partial square sequence on a stationary subset of $\aleph_{\omega+1} \cap \operatorname{cof}\left(\omega_{1}\right)$ and that $\aleph_{\omega}^{\aleph_{0}}=\aleph_{\omega+1}$. Then there is a stationary set $R \subset[H(\lambda)]^{\aleph_{0}}$ such that if $X$ is an elementary substructure of $\left\langle H(\lambda), \in, \Delta, \mathfrak{A}_{0}\right\rangle, \omega_{1} \subset X$, and $\operatorname{cof}\left(X \cap \aleph_{\alpha}\right)$ has uncountable cofinality for all $2 \leq \alpha \in \omega$ and $\alpha=\omega+1$, and $X$ reflects $R$, then $X \cap[X]^{\aleph_{0}}$ is stationary.

(We note that the hypotheses of the theorem do not require that $X$ have cardinality $\omega_{1}$, though this is the main application. Also, all that is used in the proof, is that $\sup \left(p c f\left\{\aleph_{n}: n \in \omega\right\}\right)=\aleph_{\omega+1}$. Following methods of [5] one can eliminate that assumption, assuming that partial square sequences exist on all cardinals.)

Proof. Let $R$ be the collection of reconstructible sets. We note that every element $N$ of $R$ can be canonically defined by the parameters:

(1) $\delta=\sup \left(N \cap \aleph_{\omega+1}\right)$

(2) the least $k, \chi_{N}={ }_{k} f_{\delta}$, and

(3) $\left\langle\sup \left(N \cap \omega_{k}\right), \sup \left(N \cap \omega_{k-1}\right), \ldots, N \cap \omega_{1}\right\rangle$

simply by taking the Skolem hull in $\mathfrak{A}_{0}$ of $C_{\delta}$ together with the ladder systems attached to each $\sup \left(N \cap \omega_{m}\right),(1<m \leq k)$ and $N \cap \omega_{1}$.

Suppose now that $X$ is an uncountable set including all of the countable ordinals that satisfies the cofinality restriction and $X \prec H(\lambda)$. Then $X \cap \aleph_{\omega+1}$ is $\omega$-closed. Hence if $N \subset X$ belongs to $R$, the finitely many parameters needed to define $N$ belong to $X$. Hence $N \in X$.

Corollary 42. Assume that there is a partial square on a stationary subset of $\aleph_{\omega+1} \cap \operatorname{cof}\left(\omega_{1}\right)$. Suppose that any four stationary sets simultaneously reflect. Then every stationary subset of $\left[H\left(\aleph_{\omega+1}\right)\right]^{\aleph_{0}}$ reflects to an internally stationary set. In particular, if the $\mathrm{CH}$ holds, then every stationary set reflects to an internally approachable set.

Proof. As in Corollary 20, we fix distinct infinite subsets $r, s \subset \omega$ and consider $S_{s}$ and $S_{r}$ to be the sets that everywhere have pattern $s$ and pattern $r$ respectively. Then any $X \supset \omega_{1}$ that has cardinality $\omega_{1}$ and which reflects both $S_{s}$ and $S_{r}$ has uniform cofinality $\omega_{1}$. If $X$ also reflects the set $R$, then $X$ is internally stationary. Suppose now that we want to reflect an arbitrary set $T$. If we simultaneously reflect $T$ with $S_{r}, S_{s}$ and $R$, we get an $X$ which reflects $T$ that is internally stationary. 
(The reconstruction techniques were first investigated in [5] for uncountable structures, in the context of covering lemmas. For uncountable sets no version of square is needed.)

\section{Some QUeSTIONS}

This paper leaves many questions open. We list them below. We begin with the question that we failed to solve in this paper.

(1) Does weak reflection imply that every stationary set reflects to an internally approachable set of size $\omega_{1}$ ?

(2) Can one deduce the singular cardinal hypothesis from the weak reflection principle? This has been shown recently by Shelah.

(3) Can one deduce that there are no very good scales from the weak reflection principle?

In a different direction we would like to know if partial squares exist on successors of singular cardinals. A particular question:

(4) Does there exist a stationary set $S \subset \aleph_{\omega+1} \cap \operatorname{cof}\left(\omega_{1}\right)$ on which there is a partial square sequence?

The next few problems deal with technical issues raised in this paper that we think are of interest:

(5) Can one build a scale $\left\langle f_{\alpha}: \alpha<\aleph_{\omega+1}\right\rangle \subset \prod_{n \in a} \aleph_{n}$ (for some $a$ ) such that $T=\left\{N \prec H(\lambda): \chi_{N} \leq^{*} f_{\gamma}\right.$ where $\left.\gamma=\sup \left(N \cap \aleph_{\omega+1}\right)\right\}$ is stationary? (In this paper, we showed that such a scale exists if the SCH fails, or if there is a partial square on some stationary set of ordinals of cofinality $\omega_{1}$ in $\aleph_{\omega+1}$. Here we ask if this is a result of ZFC. Clearly if the answer to Problem 4 is affirmative, the answer to this problem is affirmative.)

(6) Letting $T$ be the set defined in the previous question, is $T_{r}=\{N \in T$ : $\left.\operatorname{pat}\left(N, \aleph_{\omega+1}\right)=r\right\}$ stationary? (Under any hypothesis?) Showing this would require combining the two main techniques of this paper.

(7) Let $X \prec H(\lambda)$ have cardinality $\omega_{1}$ and suppose that $\omega_{1} \subset X$. Then each of the following properties is implied by the later properties. Moreover, under the $\mathrm{CH}$ they are all equivalent. Are they equivalent in ZFC?

(a) $X \cap[X]^{\aleph_{0}}$ is unbounded in $[X]^{\aleph_{0}}$.

(b) $X \cap[X]^{\aleph_{0}}$ is stationary in $[X]^{\aleph_{0}}$.

(c) $X \cap[X]^{\aleph_{0}}$ is closed and unbounded in $[X]^{\aleph_{0}}$.

(d) $X$ is internally approachable.

(8) Suppose that $X \prec H(\lambda)$ has cardinality $\omega_{1}$ and uniform cofinality $\omega_{1}$. Let $\kappa$ be a regular cardinal. Suppose that $X \cap[X \cap \kappa]^{\aleph_{0}}$ is stationary. Is it true that $X \cap\left[X \cap \kappa^{+}\right]^{\aleph_{0}}$ is stationary?

(9) Suppose for all stationary sets $S \subset[H(\lambda)]^{\aleph_{0}}$ there is a set $X$ of cardinality $\omega_{1}$ such that $S \cap[X]^{\aleph_{0}}$ is stationary. Is it true that for all stationary sets $S \subset[H(\lambda)]^{\aleph_{0}}$ there is such an $X$ with $\omega_{1} \subset X$ ?

\section{REFERENCES}

[1] Lev Bukovský. Changing cofinality of $\aleph_{2}$. In Set theory and hierarchy theory (Proc. Second Conf., Bierutowice, 1975), pp. 37-49. Lecture Notes in Math., Vol. 537. Springer, Berlin, 1976. MR55:5441

[2] Lev Bukovský and Eva Copláková-Hartová. Minimal collapsing extensions of models of zfc. Ann. Pure Appl. Logic, 46(3):265-298, 1990. MR92e:03077 
[3] Maxim R. Burke and Menachem Magidor. Shelah's PCF theory and its applications. Ann. Pure Appl. Logic, 50(3):207-254, 1990. MR92f:03053

[4] James Cummings, Matthew Foreman, and Menachem Magidor. Squares, scales and stationary reflection. J. Math. Log. 1(1):35-98, 2001. MF 2003a:03068

[5] James Cummings, Matthew Foreman, and Menachem Magidor. Canonical structure in the universe of set theory, parts I and II. To Appear in J. Pure Appl. Logic.

[6] M. Foreman, M. Magidor, and S. Shelah. Martin's maximum, saturated ideals, and nonregular ultrafilters. I. Ann. of Math. (2), 127(1):1-47, 1988. MR89f:03043

[7] Matthew Foreman. Stationary sets, Chang's conjecture and Partition theory. In Set Theory: The Hajnal Conference. DIMACS Ser. Discrete Math. Theoret. Comput. Sci., 58, Amer. Math. Soc., Providence, RI, 2002, pp. 73-94. MR2003e:03089

[8] Matthew Foreman and Menachem Magidor. Mutually stationary sets and the saturation of the non-stationary ideal on $P_{\kappa}(\lambda)$. Acta Math., 186(2):271-300, 2001. MR.2002g:03094

[9] Matthew Foreman and Menachem Magidor. Large cardinals and definable counterexamples to the continuum hypothesis. Ann. Pure Appl. Logic, 76(1):47-97, 1995. MF 96k:03124

[10] Matthew Foreman and Menachem Magidor. A very weak square principle. J. Symbolic Logic, 62(1):175-196, 1997. MR98i:03062

[11] Fred Galvin and András Hajnal. Inequalities for cardinal powers. Ann. of Math. (2), 101:491498, 1975. MR51:12535

[12] Thomas Jech. Singular cardinal problem: Shelah's theorem on $2^{\aleph_{\omega}}$. Bull. London Math. Soc., 24(2):127-139, 1992. MR93a:03050

[13] Kanji Namba. Independence proof of $\left(\omega, \omega_{\alpha}\right)$-distributive law in complete Boolean algebras. Comment. Math. Univ. St. Paul., 19:1-12, 1971. MR 45:6602

[14] Kanji Namba. $\left(\omega_{1}, 2\right)$-distributive law and perfect sets in generalized Baire space. Comment. Math. Univ. St. Paul., 20:107-126, 1971/72. MR 45:8593

[15] Matatyahu Rubin and Saharon Shelah. Combinatorial problems on trees: partitions, $\delta$ systems and large free subtrees. Ann. Pure Appl. Logic, 33(1):43-81, 1987. MR88h:04005

[16] Stewart Shapiro (ed.) The limits of logic: Second order logic and the Skolem paradox. The international research library of philosophy. Dartmouth Publishing Company, 1996.

[17] Saharon Shelah. Proper forcing. Springer-Verlag, Berlin, 1982. MR84h:03002

[18] Saharon Shelah. Cardinal arithmetic. The Clarendon Press, Oxford University Press, New York, 1994. Oxford Science Publications. MR.96e:03001

[19] Saharon Shelah. On what I do not understand (and have something to say). I. Fund. Math., 166(1-2):1-82, 2000. Saharon Shelah's anniversary issue. MR.2002a:03091

[20] Jack Silver. On the singular cardinals problem. In Proceedings of the International Congress of Mathematicians (Vancouver, B.C., 1974), Vol. 1, pp. 265-268. Canad. Math. Congress, Montreal, Que., 1975. MR 55:2576

[21] Thoralf Skolem. Logisch-kombinatorische Untersuchungen über die Erfüllbarkeit oder Beweisbarkeit mathematischer Sätze nebst einem Theoreme über dichte Mengen. Skrifter utgit av Videnskabsselskapet i Kristiania, I. Matematisk-naturvidenskabelig klasses, 4. 1920.

[22] Stevo Todorčević. Conjectures of Rado and Chang and cardinal arithmetic. In Finite and infinite combinatorics in sets and logic (Banff, AB, 1991), pp. 385-398. Kluwer Acad. Publ., Dordrecht, 1993. MF.95h:03113

[23] Boban Veličković. Forcing axioms and stationary sets. Adv. Math., 94(2):256-284, 1992. MR 93k:03045

[24] W. Hugh Woodin. The axiom of determinacy, forcing axioms, and the nonstationary ideal. Walter de Gruyter \& Co., Berlin, 1999. MR.2001e:03001

Department of Mathematics, University of California, Irvine, California 92697

E-mail address: mforeman@math.uci.edu

CNRS, Université Paris Vit, 2 Place Jussieu, 75251 Paris Cedex 05, Paris, France

E-mail address: stevo@logique.jussieu.fr 